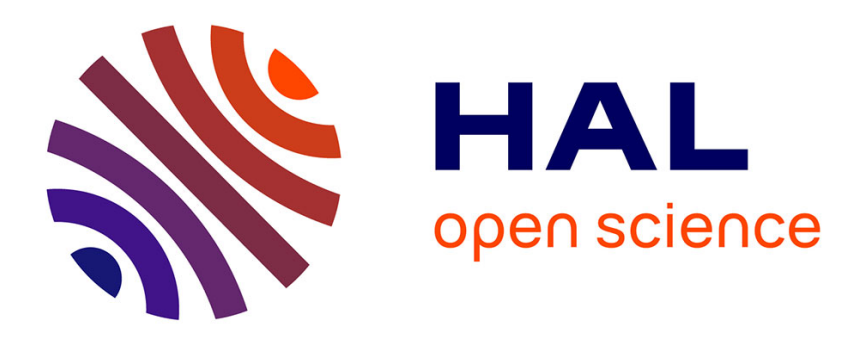

\title{
Principal eigenvalues and the Dirichlet problem for fully nonlinear operators
}

Alexander Quaas, Boyan Sirakov

\section{To cite this version:}

Alexander Quaas, Boyan Sirakov. Principal eigenvalues and the Dirichlet problem for fully nonlinear operators. Advances in Mathematics, 2008, 218 (1), pp.105-135. hal-00004692v3

\section{HAL Id: hal-00004692 \\ https://hal.science/hal-00004692v3}

Submitted on 9 Jan 2009

HAL is a multi-disciplinary open access archive for the deposit and dissemination of scientific research documents, whether they are published or not. The documents may come from teaching and research institutions in France or abroad, or from public or private research centers.
L'archive ouverte pluridisciplinaire HAL, est destinée au dépôt et à la diffusion de documents scientifiques de niveau recherche, publiés ou non, émanant des établissements d'enseignement et de recherche français ou étrangers, des laboratoires publics ou privés. 


\title{
Principal eigenvalues and the Dirichlet problem for fully nonlinear elliptic operators
}

\author{
Alexander QUAAS ${ }^{1}$ \\ Departamento de Matemática, Universidad Santa Maria \\ Avenida España 1680, Casilla 110-V, Valparaíso, Chile \\ Boyan SIRAKOV ${ }^{2}$ \\ UFR SEGMI, Université Paris 10, 92001 Nanterre Cedex, France \\ and CAMS, EHESS, 54 bd Raspail, 75270 Paris Cedex 06, France
}

\begin{abstract}
We study uniformly elliptic fully nonlinear equations of the type $F\left(D^{2} u, D u, u, x\right)=f(x)$. We show that convex positively 1-homogeneous operators possess two principal eigenvalues and eigenfunctions, and study these objects ; we obtain existence and uniqueness results for non-proper operators whose principal eigenvalues (in some cases, only one of them) are positive ; finally, we obtain an existence result for non-proper Isaac's equations.
\end{abstract}

2000 Mathematics Subject Classification : 35J60, 35J65, 35P30.

\section{Introduction and Main Results}

This paper is a study of uniformly elliptic fully nonlinear equations in nondivergence form

$$
F\left(D^{2} u, D u, u, x\right)=f(x)
$$

in a bounded domain $\Omega \subset \mathbb{R}^{N}$. We pursue the following goals. First, we show that positively homogeneous operators which are convex (or concave) - like Hamilton-Jacobi-Bellman operators, that is, suprema of linear operators with non-smooth coefficients - possess two principal eigenvalues, corresponding to a positive and a negative principal eigenfunction, and study properties of these objects. Second, we show that existence and uniqueness theory can be developed for coercive non-proper operators, more precisely, for operators whose both principal eigenvalues (or, in some cases, only one of them) are positive. Finally, we obtain existence results for the Dirichlet problem for non-proper operators which are not convex (like Isaac's equations, that is, sup-inf of linear operators), under the hypothesis that some related operator is coercive.

\footnotetext{
${ }^{1}$ Supported by FONDECYT, Grant N. 1040794, and ECOS grant C02E08.

${ }^{2}$ Corresponding author, e-mail : sirakov@ehess.fr
} 
It has long been known that certain types of positively homogeneous operators possess "half"-eigenvalues. The first to observe this phenomenon was Berestycki in [Be], where he considered bifurcation for some Sturm-Liouville problems. An important step in studying these types of questions was made by Lions [L1]. By combining probability and analytical methods, in that paper he proved the existence of principal eigenvalues for operators which are the supremum of linear operators with $C^{1,1}$-coefficients, and obtained results about the solvability of related Dirichlet problems. Recently the question of existence of principal eigenvalues was addressed in another particular case, namely when $F$ is a Pucci extremal operator $\left(\mathcal{M}_{\lambda, \Lambda}^{+}\left(D^{2} u\right)\right.$, see below), by Felmer and Quaas [FQ] (see also [BEQ], [Q]). Related results were obtained for operators of the type $|D u|^{\alpha} \mathcal{M}_{\lambda, \Lambda}^{+}\left(D^{2} u\right)$ by Birindelli and Demengel [BD1]. The results in all the quoted papers are partial in the sense that many known properties of the principal eigenvalue of a linear operator were left open. These properties include the facts that the eigenvalue is simple, isolated, and its positivity is necessary and sufficient for the operator to satisfy comparison principle, for the Alexandrov-Bakelman-Pucci inequality to hold, and to guarantee that the Dirichlet problem is solvable for any right-hand side. It is our aim here to bring the eigentheory of fully nonlinear equations closer to the level of the well studied linear case, for which we refer to the paper by Berestycki, Nirenberg and Varadhan $[\mathrm{BNV}]$ - a deep study of properties of the principal eigenvalue of linear elliptic operators in non-divergence form, as well as of related maximum principles and existence theory for strong solutions of linear equations. The results we obtain extend most of the main results in $[\mathrm{BNV}]$ to nonlinear operators and viscosity solutions, and exhibit the differences due to the nonlinear nature of the operators we consider.

In the last thirty years there have been a multitude of results on existence, uniqueness and regularity properties of classical, strong or viscosity solutions of equations of type (1.1). For the classical case we refer to the works [L2], [E], $[\mathrm{Bu}]$, and to the books $[\mathrm{K}],[\mathrm{GT}]$. For strong solutions of linear equations, see [GT], Chapter 9. As far as viscosity solutions are concerned, we shall quote here the fundamental work [CIL], where very general equations are studied, as well as [CC], [CCKS], [CKLS], [JS], [S] (see also the references in these works), where a more specific - and close to ours - setting is considered. Typical structure conditions on $F$ assumed in these papers are

$$
\begin{aligned}
\mathcal{M}_{\lambda, \Lambda}^{-}(M-N)-\gamma|p-q| & \leq F(M, p, u, x)-F(N, q, u, x) \\
& \leq \mathcal{M}_{\lambda, \Lambda}^{+}(M-N)+\gamma|p-q|
\end{aligned}
$$

for some positive constants $\lambda, \Lambda, \gamma$ and any $M, N \in \mathcal{S}_{N}$ (the set of all $N \times N$ symmetric matrices), $p, q \in \mathbb{R}^{N}, u \in \mathbb{R}, x \in \Omega$, and

$(P) \quad F$ is proper, that is, $F$ is nonincreasing in $u$. 
Recall that Pucci's operators are defined by $\mathcal{M}_{\lambda, \Lambda}^{+}(M)=\sup _{A \in \mathcal{A}} \operatorname{tr}(A M)$, $\mathcal{M}_{\lambda, \Lambda}^{-}(M)=\inf _{A \in \mathcal{A}} \operatorname{tr}(A M)$, where $\mathcal{A} \subset \mathcal{S}_{N}$ denotes the set of matrices whose eigenvalues lie in the interval $[\lambda, \Lambda]$. Note $\mathcal{M}_{\lambda, \Lambda}^{+}(M)=-\mathcal{M}_{\lambda, \Lambda}^{-}(-M)$.

Condition $(\mathrm{P})$ is too restrictive compared to what is known for linear operators. For instance, consider the linear operator $L=\operatorname{tr}\left(A D^{2} \cdot\right)+c(x)$. It is well known that the Dirichlet problem for $L u=f \in L^{N}$ is uniquely solvable provided $\lambda_{1}(L)>0$. On the other hand, if $\operatorname{tr}\left(A D^{2} \cdot\right)$ is replaced by a nonlinear operator satisfying $(S)$, all the above quoted papers concern the case $c(x) \leq 0$. We will show that for a nonlinear operator the right hypothesis under which the Dirichlet problem is solvable for any right-hand side is again the positivity of the principal eigenvalues.

The paper is organized as follows. In the next section we give assumptions and define the principal eigenvalues. In Sections 1.2 and 1.3 we state our main results, about properties of principal eigenvalues and eigenfunctions, and about solvability of the Dirichlet problem. In Section 2 we give some important examples of operators to which our results apply, and discuss different situations that can arise. An intriguing difference between the two eigenvalues is put into light. Finally, in Section 3 we recall some previous results which we use, and in Section 4 we give the proofs of our results.

\subsection{Assumptions and definition of $\lambda_{1}^{+}, \lambda_{1}^{-}$}

The operator $F$ is supposed to be defined on $\mathcal{S}_{N} \times \mathbb{R}^{N} \times \mathbb{R} \times(\Omega \backslash \mathcal{N})$, where $\mathcal{N} \subset \Omega$ is a null set.

Next, we assume that the operator is positively homogeneous of order 1 , that is,

$\left(H_{0}\right) \quad F(t M, t p, t u, x)=t F(M, p, u, x), \quad$ for all $t \geq 0$.

Everywhere in the sequel we consider operators which satisfy the following hypothesis : for some $\gamma, \delta>0$ and all $M, N \in \mathcal{S}_{N}, p, q \in \mathbb{R}^{N}, u, v \in \mathbb{R}, x \in \Omega$, $\left(H_{1}\right)$

$$
\mathcal{M}_{\lambda, \Lambda}^{-}(M-N)-\gamma|p-q|-\delta|u-v| \leq F(M, p, u, x)-F(N, q, v, x)
$$

$$
\leq \mathcal{M}_{\lambda, \Lambda}^{+}(M-N)+\gamma|p-q|+\delta|u-v|
$$

We are going to suppose that

$$
F(M, 0,0, x) \text { is continuous in } \mathcal{S}_{N} \times \bar{\Omega} \text {. }
$$

Note that when $F$ is linear $\left(H_{1}\right)-\left(H_{2}\right)$ mean $F$ is uniformly elliptic, with bounded coefficients, and continuous second-order coefficients.

We denote

$$
G(M, p, u, x)=-F(-M,-p,-u, x) .
$$


An important role will be played by the following definition. We say that an operator $H(M, p, u, x)$ satisfies condition $\left(D_{F}\right)$ provided

$$
\begin{aligned}
G(M-N, p-q, u-v, x) & \leq H(M, p, u, x)-H(N, q, v, x) \\
& \leq F(M-N, p-q, u-v, x) .
\end{aligned}
$$

This hypothesis permits to measure how far an operator $H$ is from linear the extremes of $\left(D_{F}\right)$ are attained, on one hand, when $F$ is linear (so that $F=G$ and $H(M, p, u, x)=F(M, p, u, x)+H(0,0,0, x))$, and on the other hand, when $F(M, p, u, x)=\mathcal{M}_{\lambda, \Lambda}^{+}(M)+\gamma|p|+\delta|u|-$ then $\left(D_{F}\right)$ reduces to $\left(H_{1}\right)$.

Lemma 1.1 Suppose $F$ satisfies $\left(H_{0}\right)$. Then the following are equivalent

(i) F is convex in $(M, p, u)$; (ii) F satisfies $\left(D_{F}\right)$;

(iii) F satisfies one of the two inequalities in $\left(D_{F}\right)$.

We shall assume that the domain $\Omega$ is smooth. We stress however that most results can be extended to arbitrary bounded domains, by using an approximation argument, as in [BNV], see Section 5.

We make the convention that each time we use the term viscosity solution we mean $L^{N}$-viscosity - see for example [CCKS] for definitions and properties of these. Also, any time we say a non-regular function satisfies an (in)equality, we shall mean it is satisfied in the viscosity sense.

For any $\lambda \in \mathbb{R}$ we define the sets

$\Psi^{+}(F, \Omega, \lambda)=\left\{\psi \in C(\bar{\Omega}) \mid \psi>0\right.$ in $\Omega, F\left(D^{2} \psi, D \psi, \psi, x\right)+\lambda \psi \leq 0$ in $\left.\Omega\right\}$, $\Psi^{-}(F, \Omega, \lambda)=\left\{\psi \in C(\bar{\Omega}) \mid \psi<0\right.$ in $\Omega, F\left(D^{2} \psi, D \psi, \psi, x\right)+\lambda \psi \geq 0$ in $\left.\Omega\right\}$, and the following (finite, see Proposition 4.2) quantities

$$
\begin{aligned}
& \lambda_{1}^{+}(F, \Omega)=\sup \left\{\lambda \mid \Psi^{+}(F, \Omega, \lambda) \neq \emptyset\right\}, \\
& \lambda_{1}^{-}(F, \Omega)=\sup \left\{\lambda \mid \Psi^{-}(F, \Omega, \lambda) \neq \emptyset\right\} .
\end{aligned}
$$

We shall not write the dependence of $\lambda_{1}^{+}, \lambda_{1}^{-}$in $\Omega$ or in $F$, when no confusion arises. Note that $\lambda_{1}^{+}(F)=\lambda_{1}^{-}(G)$ and $\lambda_{1}^{-}(F)=\lambda_{1}^{+}(G)$. Note also that $\lambda_{1}^{+}(F) \leq \lambda_{1}^{-}(F)$ since $F$ is convex. We will show later that the sets $\Psi$ in the definitions of $\lambda_{1}^{+}, \lambda_{1}^{-}$can be replaced by much smaller ones - see (4.16) and Proposition 4.11.

Next, we turn to the statements of our results. In order to facilitate the task of the reader acknowledged with the corresponding results for linear operators, we have adopted a way of exposing similar to the one in [BNV] 
- we believe this better highlights similarities and differences. It should be stressed however that our proofs, while borrowing some ideas from the linear case, require a different overall approach, and that essential points in the proofs are very nonlinear in nature - for example the construction of a supersolution in Proposition 4.12. We give an overview in Section 4.

\subsection{Existence and properties of eigenvalues}

The following theorem asserts the existence of two pairs of principal eigenfunctions and eigenvalues of a nonlinear operator. Set $E_{p}=W_{\mathrm{loc}}^{2, p}(\Omega) \cap C(\bar{\Omega})$.

Theorem 1.1 Suppose $F$ satisfies $\left(H_{0}\right),\left(H_{1}\right),\left(H_{2}\right)$, and $\left(D_{F}\right)$. Then there exist functions $\varphi_{1}^{+}, \varphi_{1}^{-} \in E_{p}$ for all $p<\infty$, such that

$$
\left\{\begin{aligned}
F\left(D^{2} \varphi_{1}^{+}, D \varphi_{1}^{+}, \varphi_{1}^{+}, x\right) & =-\lambda_{1}^{+} \varphi_{1}^{+} & & \text {in } \Omega \\
\varphi_{1}^{+} & >0 & & \text { in } \Omega \\
\varphi_{1}^{+} & =0 & & \text { on } \partial \Omega
\end{aligned}\right.
$$

and

$$
\left\{\begin{aligned}
F\left(D^{2} \varphi_{1}^{-}, D \varphi_{1}^{-}, \varphi_{1}^{-}, x\right) & =-\lambda_{1}^{-} \varphi_{1}^{-} & & \text {in } \Omega \\
\varphi_{1}^{-} & <0 & & \text { in } \Omega \\
\varphi_{1}^{-} & =0 & & \text { on } \partial \Omega .
\end{aligned}\right.
$$

If $\varphi_{1}^{+}$(or $\left.\varphi_{1}^{-}\right)$is normalized so that $\varphi_{1}^{+}\left(x_{0}\right)=1$ (resp. $\varphi_{1}^{-}\left(x_{0}\right)=-1$ ) for a fixed point $x_{0} \in \Omega$, then $\varphi_{1}^{+} \leq C$ (resp. $\varphi_{1}^{-} \geq-C$ ) in $\Omega$, where $C$ depends only on $x_{0}, \Omega, \lambda, \Lambda, \gamma$ and $\delta$.

In addition, $\lambda_{1}^{+}$(resp. $\lambda_{1}^{-}$) is the only eigenvalue corresponding to a positive (resp. negative) eigenfunction in $C(\bar{\Omega})$.

The next result implies that the principal eigenfunctions are simple in a strong sense, even in the set of viscosity solutions.

Theorem 1.2 Suppose F satisfies $\left(H_{0}\right),\left(H_{1}\right),\left(H_{2}\right)$, and $\left(D_{F}\right)$. Assume there exists a viscosity solution $u \in C(\bar{\Omega})$ of

$$
\left\{\begin{aligned}
F\left(D^{2} u, D u, u, x\right) & =-\lambda_{1}^{+} u & & \text { in }
\end{aligned}\right.
$$

or of

$$
\left\{\begin{array}{l}
F\left(D^{2} u, D u, u, x\right) \geq-\lambda_{1}^{+} u \text { in } \Omega \\
u\left(x_{0}\right)>0, \quad \text { on } \partial \Omega
\end{array}\right.
$$

for some $x_{0} \in \Omega$. Then $u \equiv t \varphi_{1}^{+}$, for some $t \in \mathbb{R}$. If a function $v \in C(\bar{\Omega})$ satisfies either (1.2) or the reverse inequalities in (1.3), with $\lambda_{1}^{+}$replaced by $\lambda_{1}^{-}$, then $v \equiv t \varphi_{1}^{-}$for some $t \in \mathbb{R}$. 
Remark. Taking $u=\varphi_{1}^{-}$shows $u\left(x_{0}\right)>0$ cannot be removed from (1.3).

The following theorem states that the eigenvalues are isolated.

Theorem 1.3 Suppose $F$ satisfies $\left(H_{0}\right),\left(H_{1}\right),\left(H_{2}\right)$, and $\left(D_{F}\right)$. There exists $\varepsilon_{0}>0$ depending on $N, \Omega, \lambda, \Lambda, \gamma, \delta$, such that the problem

$$
\left\{\begin{aligned}
F\left(D^{2} u, D u, u, x\right) & =-\lambda u & \text { in } & \Omega \\
u & =0 & \text { on } & \partial \Omega
\end{aligned}\right.
$$

has no solutions $u \not \equiv 0$, for $\lambda \in\left(-\infty, \lambda_{1}^{-}+\varepsilon_{0}\right) \backslash\left\{\lambda_{1}^{+}, \lambda_{1}^{-}\right\}$.

It is an interesting question whether the two eigenvalues can coincide for truly nonlinear operators, and, when they do not, whether the eigenfunctions can or may not differ only by a multiplication by a constant. The answers are all affirmative, see Section 2.

The next theorem gives a necessary and sufficient condition for the positivity of the principal eigenvalues. It also shows that the existence of a positive viscosity supersolution implies the existence of a positive uniformly bounded (below, and in the global $W^{2, p}$-norm) strong supersolution.

Theorem 1.4 Suppose $F$ satisfies $\left(H_{0}\right),\left(H_{1}\right),\left(H_{2}\right)$, and $\left(D_{F}\right)$.

(a) Assume there is a function $u \in C(\bar{\Omega})$ such that

$$
\left\{\begin{array}{r}
F\left(D^{2} u, D u, u, x\right) \leq 0 \text { in } \Omega \\
u
\end{array}\right.
$$

in the viscosity sense. Then either $\lambda_{1}^{+}>0$ or $\lambda_{1}^{+}=0$ with $u \equiv t \varphi_{1}^{+}$, for some $t>0$ (resp. $\lambda_{1}^{-}>0$ or $\lambda_{1}^{-}=0$ with $u \equiv t \varphi_{1}^{-}$, for some $\left.t>0\right)$.

(b) Conversely, if $\lambda_{1}^{+}>0$ then there exists a function $u \in W^{2, p}(\Omega)$, $p<\infty$, such that $F\left(D^{2} u, D u, u, x\right) \leq 0, u \geq 1$ in $\Omega$, and $\|u\|_{W^{2, p}(\Omega)} \leq C$, where $C$ depends on $p, N, \Omega, \lambda, \Lambda, \gamma, \delta$, and $\lambda_{1}^{+}$.

Remark 1. When $F$ is proper, $u \equiv 1$ satisfies the condition of Theorem 1.4. Hence proper operators have positive eigenvalues.

Remark 2. Propositions 4.2 and 4.8 provide upper and lower bounds (in terms of $F$ and $\Omega$ ) for $\lambda_{1}^{+}$and $\lambda_{1}^{-}$.

Remark 3. It follows from Theorem 1.4 that the eigenvalues are strictly decreasing with respect to the domain : if $\Omega \varsubsetneqq \Omega^{\prime}$ then $\lambda_{1}^{+}(\Omega)>\lambda_{1}^{+}\left(\Omega^{\prime}\right)$ and $\lambda_{1}^{-}(\Omega)>\lambda_{1}^{-}\left(\Omega^{\prime}\right)$ (take $u=\varphi_{1}^{+}\left(\Omega^{\prime}\right)$ in Theorem 1.4). Note that $\lambda_{1}^{+}$and $\lambda_{1}^{-}$ are continuous with respect to the domain, as Proposition 4.10 shows.

Further, we show that, similarly to the linear case, the positivity of the principal eigenvalues is a necessary and sufficient condition for the operator 
to satisfy a comparison principle. We say that a second order operator $H$ satisfies a comparison principle $(\mathrm{CP})$, provided for any $u, v \in C(\bar{\Omega})$, one of which is in $E_{N}$, such that

$$
\left\{\begin{array}{rlrl}
H\left(D^{2} u, D u, u, x\right) & \geq H\left(D^{2} v, D v, v, x\right) & & \text { in } \Omega \\
u & \leq v & \text { on } \partial \Omega
\end{array}\right.
$$

we have $u \leq v$ in $\Omega$. A particular case of $(\mathrm{CP})$ is the maximum principle, when one of $u, v$ is set to zero (and $H(0,0,0, x) \equiv 0$, as we always assume).

Theorem 1.5 Suppose a second-order operator $F$ satisfies $\left(H_{0}\right),\left(H_{1}\right),\left(H_{2}\right)$, and $\left(D_{F}\right)$. Then $\lambda_{1}^{+}(F)>0$ is necessary and sufficient for $F$ to satisfy $(C P)$. Hence, if a second-order operator $H$ satisfies $\left(D_{F}\right)$, then $\lambda_{1}^{+}(F)>0$ is sufficient for $H$ to satisfy $(C P)$.

If $\lambda_{1}^{-}>0$, the comparison and even the maximum principle do not necessarily hold. However, it can be shown that $\lambda_{1}^{-}>0$ is necessary and sufficient for a one-sided maximum principle.

Theorem 1.6 Suppose a second-order operator $F$ satisfies $\left(H_{0}\right),\left(H_{1}\right),\left(H_{2}\right)$, and $\left(D_{F}\right)$. Then $F$ has the following property : for any $u \in C(\bar{\Omega})$,

$$
\left\{\begin{aligned}
F\left(D^{2} u, D u, u, x\right) & \leq 0 \text { in } \Omega \\
u & \geq 0 \text { on } \partial \Omega
\end{aligned} \quad \text { implies } u \geq 0 \text { in } \Omega,\right.
$$

if and only if $\lambda_{1}^{-}(F)>0$.

Finally, we have the following Alexandrov-Bakelman-Pucci (ABP) inequality for nonproper second order operators. The ABP inequality is an essential tool in studying non-divergence form equations - see [GT], [BNV], [CCKS] for discussions.

Theorem 1.7 Suppose the operator F satisfies $\left(H_{0}\right),\left(H_{1}\right),\left(H_{2}\right)$, and $\left(D_{F}\right)$. Then for any $u \in C(\bar{\Omega}), f \in L^{N}(\Omega)$, the inequalities $F\left(D^{2} u, D u, u, x\right) \geq f$, $\lambda_{1}^{+}(F)>0$ (resp. $F\left(D^{2} u, D u, u, x\right) \leq f, \lambda_{1}^{-}(F)>0$ ) imply

$\sup _{\Omega} u \leq C\left(\sup _{\partial \Omega} u^{+}+\left\|f^{-}\right\|_{L^{N}(\Omega)}\right), \quad\left(\operatorname{resp} . \sup _{\Omega} u^{-} \leq C\left(\sup _{\partial \Omega} u^{-}+\left\|f^{+}\right\|_{L^{N}(\Omega)}\right)\right)$.

where $C$ depends on $\Omega, N, \lambda, \Lambda, \gamma, \delta$, and $\lambda_{1}^{+}(F)$ (resp. $\left.\lambda_{1}^{-}(F)\right)$. 


\subsection{The Dirichlet problem}

We will show that the Dirichlet problem for our type of operators is solvable for any right-hand side if the eigenvalues of the operator are positive, and that the Dirichlet problem may not be solvable if only one of them is positive.

Theorem 1.8 Suppose $F$ satisfies $\left(H_{0}\right),\left(H_{1}\right),\left(H_{2}\right)$, and $\left(D_{F}\right)$. If $\lambda_{1}^{+}(F)>0$ then for any $f \in L^{p}(\Omega), p \geq N$, there exists a unique solution $u \in E_{p}$ of

$$
\left\{\begin{aligned}
F\left(D^{2} u, D u, u, x\right) & =f \text { in } \quad \Omega \\
u & =0 \text { on } \partial \Omega
\end{aligned}\right.
$$

In addition, for any compact set $\omega \subset \subset \Omega$ there holds

$$
\|u\|_{W^{2, p}(\omega)} \leq C\|f\|_{L^{p}(\Omega)},
$$

where $C$ depends on $p, \omega, \Omega, \lambda, \Lambda, \gamma, \delta$, and $\lambda_{1}^{+}(F)$.

On the other hand, if $\lambda_{1}^{+}(F)=0$ or $\lambda_{1}^{-}(F)>0 \geq \lambda_{1}^{+}(F)$ then problem (1.5) does not possess a solution in $C(\bar{\Omega})$, provided $f \leq 0, f \not \equiv 0$ in $\Omega$.

Remark. In $[\mathrm{Bu}]$ Busca showed (through different techniques) the existence of a unique classical solution of the Dirichlet problem in the particular case when $F$ is a supremum of a countable family of linear operators with Hölder continuous coefficients and uniformly positive first eigenvalues. This uniform positivity turns out to be equivalent to $\lambda_{1}^{+}>0$, as our results show (see the next section).

As Theorem 1.8 shows, if only one of the two eigenvalues is positive, the Dirichlet problem may not have a solution. However, it still does provided the right-hand side is nonnegative.

Theorem 1.9 Suppose $F$ satisfies $\left(H_{0}\right),\left(H_{1}\right),\left(H_{2}\right),\left(D_{F}\right)$. If $\lambda_{1}^{-}(F)>0$ then for any $f \in L^{p}(\Omega), p \geq N$, such that $f \geq 0$ in $\Omega$, there exists a nonpositive solution $u \in E_{p}$ of (1.5).

Remark. We do not know if the solution obtained in Theorem 1.9 is unique.

As is known, the problem of solvability and uniqueness for the Dirichlet problem for non-convex operators is quite complicated. The following existence result, applicable to Isaac's operators, completes (and uses) some recent results for proper operators, obtained in [CKLS] (see also [JS]). It says an Isaac's equation is solvable provided the operator is controlled, in the sense of $\left(D_{F}\right)$, by an operator with positive eigenvalues. 
Theorem 1.10 Assume $F$ satisfies $\left(H_{0}\right),\left(H_{1}\right),\left(H_{2}\right),\left(D_{F}\right)$, and $H$ satisfies $\left(D_{F}\right)$ and $\left(H_{0}\right)$. If $\lambda_{1}^{+}(F)>0$ then the problem

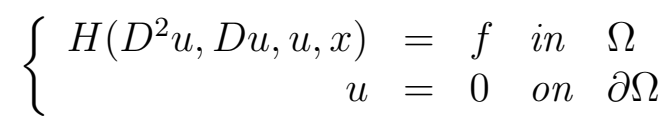

is solvable in the viscosity sense for any $f \in L^{p}(\Omega), p \geq N$. If $H(M, p, u, x)$ is convex in $M$ then $u \in E_{p}$, and $u$ is unique.

\section{Examples and Discussion}

We have proved the existence of principal eigenvalues of Hamilton-JacobiBellman operators

$$
\begin{gathered}
F_{H J B}\left(D^{2} u, D u, u, x\right)=\sup _{\alpha \in \mathcal{A}} L_{x}^{\alpha} u, \quad \text { with } \\
L_{x}^{\alpha} u:=\operatorname{tr}\left(A^{\alpha}(x) D^{2} u\right)+\vec{b}^{\alpha}(x) \cdot D u+c^{\alpha}(x) u,
\end{gathered}
$$

where $\mathcal{A}$ is an arbitrary index set, $A^{\alpha}(x)$ are matrices which depend continuously on $x$ and such that $\lambda I \leq A^{\alpha}(x) \leq \Lambda I$, and $\vec{b}^{\alpha}: \mathbb{R}^{N} \rightarrow \mathbb{R}^{N}, c^{\alpha}: \mathbb{R}^{N} \rightarrow \mathbb{R}$ are measurable bounded (uniformly in $\alpha$ ) functions.

Note that the question of existence and uniqueness of solutions of Hamilton-Jacobi-Bellman equations is often set in the form : given a family of linear operators as above and a family of continuous functions $f^{\alpha}$, solve

$$
\sup _{\alpha \in \mathcal{A}}\left(\operatorname{tr}\left(A^{\alpha}(x) D^{2} u\right)+\vec{b}^{\alpha}(x) \cdot D u+c^{\alpha}(x) u-f^{\alpha}(x)\right)=0 .
$$

This equation is in the form $H\left(D^{2} u, D u, u, x\right)=f(x)$, where

$$
H\left(D^{2} u, D u, u, x\right)=\sup _{\alpha \in \mathcal{A}}\left(L_{x}^{\alpha} u-f^{\alpha}(x)\right)+\inf _{\alpha \in \mathcal{A}} f^{\alpha}(x), \quad f(x)=\inf _{\alpha \in \mathcal{A}} f^{\alpha}(x) .
$$

This $H$ is convex in $(M, p, u)$ and satisfies $\left(D_{F_{H J B}}\right)$, so the results of the previous section apply.

It easily follows from the definitions of the eigenvalues that

$$
\lambda_{1}^{+}\left(F_{H J B}\right) \leq \inf _{\alpha \in \mathcal{A}} \lambda_{1}\left(L_{x}^{\alpha}\right) \leq \sup _{\alpha \in \mathcal{A}} \lambda_{1}\left(L_{x}^{\alpha}\right) \leq \lambda_{1}^{-}\left(F_{H J B}\right),
$$

where $\lambda_{1}\left(L_{x}^{\alpha}\right)$ denotes the usual first eigenvalue of the linear operator $L_{x}^{\alpha}$. One should clearly ask whether the first and the third inequalities in (2.6) are actually equalities. 
As far as the first inequality is concerned, it actually is an equality when the set $\mathcal{A}$ is countable, since then it is possible to show that the Dirichlet problem for $F_{H J B}$ is uniquely solvable for any right-hand side (through an argument similar to the one in $[\mathrm{Bu}])$, provided $\inf _{\alpha \in \mathcal{A}} \lambda_{1}\left(L_{x}^{\alpha}\right)>0$. Then, if the first inequality in (2.6) were strict, the operators $\widetilde{L}_{x}^{\alpha}=L_{x}^{\alpha}+\lambda_{1}^{+}\left(F_{H J B}\right)$ have uniformly positive eigenvalues but $\sup _{\alpha \in \mathcal{A}} \widetilde{L}_{x}^{\alpha} u=0$ has two solutions which vanish on $\partial \Omega$, the trivial one and $\varphi_{1}^{+}$given by Theorem 1.1 - a contradiction. Further, it is clear that the Dirichlet problem for $F_{H J B}$ remains solvable under $\inf _{\alpha \in \mathcal{A}} \lambda_{1}\left(L_{x}^{\alpha}\right)>0$, when $\mathcal{A}$ is a separable metric space such that the maps $\alpha \rightarrow A^{\alpha}, \vec{b}^{\alpha}, c^{\alpha}$ are continuous for almost every $x$ (a similar remark is made in [GT], after Theorem 17.18). This implies, for instance, that

$$
\lambda_{1}^{+}\left(\mathcal{M}_{\lambda, \Lambda}^{+}\right)=\inf _{\lambda I \leq A \leq \Lambda I} \lambda_{1}\left(\operatorname{tr}\left(A D^{2} \cdot\right)\right),
$$

the infimum being taken over constant matrices, and not over functions (as was stated in [BEQ]), which is what the mere fact that the infimum is attained suggests.

Surprisingly, it turns out that an analogous intuitive statement about the third inequality in (2.6) is wrong, as we shall show next.

First, note that $\lambda_{1}^{+}=\lambda_{1}^{-}$is possible for truly nonlinear operators. An explicit example is, for instance, $\Omega=(0, \pi) \times(0, \pi) \subset \mathbb{R}^{2}$, and

$$
F_{a, b}\left(D^{2} u\right)=\max \left\{a u_{x_{1} x_{1}}+b u_{x_{2} x_{2}}, b u_{x_{1} x_{1}}+a u_{x_{2} x_{2}}\right\}, \quad a, b>0, a \neq b,
$$

where $\varphi_{1}^{+}=-\varphi_{1}^{-}=\sin \left(x_{1}\right) \sin \left(x_{2}\right)$, and $\lambda_{1}^{+}\left(F_{a, b}\right)=\lambda_{1}^{-}\left(F_{a, b}\right)=a+b$.

Further, Theorem 1.2 (with $u=-\varphi_{1}^{-}$) easily implies that $\varphi_{1}^{+}=-\varphi_{1}^{-}$ after renormalization, whenever $\lambda_{1}^{+}=\lambda_{1}^{-}$. Let us now look at the same operator $F_{a, b}\left(D^{2} u\right)=\max \left\{L_{1} u, L_{2} u\right\}$, but on a ball $B \subset \mathbb{R}^{2}$. A simple symmetry argument shows $\lambda_{1}\left(L_{1}, B\right)=\lambda_{1}\left(L_{2}, B\right)$, so, by what we already saw, $\lambda_{1}^{+}\left(F_{a, b}\right)$ is equal to $\lambda_{1}\left(L_{1}\right)$. However, $\lambda_{1}^{-}\left(F_{a, b}\right)$ is strictly larger than $\lambda_{1}\left(L_{1}\right)=\lambda_{1}\left(L_{2}\right)$. Indeed, suppose for contradiction $\lambda_{1}^{-}=\lambda_{1}\left(L_{1}\right)=\lambda_{1}^{+}=: \lambda$. Then the principal eigenfunction $\varphi:=\varphi_{1}^{+}=-\varphi_{1}^{-}$of $F_{a, b}$ would satisfy

$$
\left\{\begin{array}{l}
F_{a, b}\left(D^{2} \varphi\right)+\lambda \varphi=0 \text { in } B \\
G_{a, b}\left(D^{2} \varphi\right)+\lambda \varphi=0 \text { in } B
\end{array}\right.
$$

By adding up the two equations in (2.7) we see that $\varphi$ is the principal eigenfunction of the Laplacian, and so $\varphi$ is smooth and radial. It also follows from (2.7) that $F_{a, b}\left(D^{2} \varphi\right)=G_{a, b}\left(D^{2} \varphi\right)$ in $B$, which means $\varphi_{x_{1} x_{1}} \equiv \varphi_{x_{2} x_{2}}$. The only smooth radial functions which satisfy this are $C_{1}|x|^{2}+C_{2}-$ a contradiction.

An example when $\lambda_{1}^{+} \neq \lambda_{1}^{-}$and $\varphi_{1}^{+}=-\varphi_{1}^{-}$is provided by the operator

$$
F_{a}\left(D^{2} u\right)=\max \{\Delta u, a \Delta u\}, \quad a>0, a \neq 1 .
$$


Then $\varphi_{1}^{+}=-\varphi_{1}^{-}$is the usual first eigenfunction of the Laplacian, however $\lambda_{1}^{+}=\min \left\{\lambda_{1}, a \lambda_{1}\right\}<\max \left\{\lambda_{1}, a \lambda_{1}\right\}=\lambda_{1}^{-}$, where $\lambda_{1}$ is the first eigenvalue of the Laplacian. Note that the spectrum of $F_{a}$ is actually the so-called Fucik spectrum of the Laplacian.

Finally, we note that $\mathcal{M}_{\lambda, \Lambda}^{+}$is an operator for which $\varphi_{1}^{+}$and $\varphi_{1}^{-}$are not proportional, for instance when $\Omega$ is a ball. Indeed, suppose for contradiction that $\varphi \in E_{p}(B)$ is a function such that $\varphi>0$ in $B, \varphi=0$ on $\partial B$, and

$$
\left\{\begin{array}{l}
\mathcal{M}_{\lambda, \Lambda}^{+}\left(D^{2} \varphi\right)+\lambda_{1}^{+} \varphi=0 \text { in } B \\
\mathcal{M}_{\lambda, \Lambda}^{-}\left(D^{2} \varphi\right)+\lambda_{1}^{-} \varphi=0 \text { in } B
\end{array}\right.
$$

for some ball $B$. By summing these two equations we obtain

$$
(\lambda+\Lambda) \Delta \varphi+\left(\lambda_{1}^{+}+\lambda_{1}^{-}\right) \varphi=0 \quad \text { in } B,
$$

so $\varphi$ is the first (radial and smooth) eigenfunction of the Laplacian.

We recall that $\mathcal{M}_{\lambda, \Lambda}^{+}(M)=\Lambda \sum_{\left\{e_{i}>0\right\}} e_{i}+\lambda \sum_{\left\{e_{i}<0\right\}} e_{i}$, where $e_{i}$ denote the eigenvalues of $M$, for any $M \in \mathcal{S}_{N}$. This, together with the fact that $\varphi$ attains its maximum at the origin, imply $\mathcal{M}_{\lambda, \Lambda}^{+}\left(D^{2} \varphi(0)\right)=\lambda \Delta \varphi(0)$. It then follows from the first equation in (2.8) that $\frac{\lambda_{1}^{+}}{\lambda}=\lambda_{1}$, where $\lambda_{1}$ is the first eigenvalue of the Laplacian.

Therefore the first equation in (2.8) reads $\mathcal{M}_{\lambda, \Lambda}^{+}\left(D^{2} \varphi\right)-\lambda \Delta \varphi=0$ in $B$, or

$$
\Lambda \sum_{\left\{e_{i}(x)>0\right\}} e_{i}(x)+\lambda \sum_{\left\{e_{i}(x)<0\right\}} e_{i}(x)-\lambda \sum_{i=1}^{N} e_{i}(x) \equiv 0 \quad \text { in } B,
$$

where $e_{i}(x)$ denote the eigenvalues of $D^{2} \varphi(x)$. Hence $e_{i}(x) \leq 0$ in $B$, for all $i=1, \ldots, N$.

However, by writing (2.9) in the radial variable and by using Hopf's lemma, it is easy to see that $D^{2} \varphi(x)$ has a positive eigenvalue in a neighbourhood of $\partial \Omega$ (see also Lemma 4.1). This is a contradiction.

Naturally, given some positive constants $\lambda, \Lambda, \gamma, \delta$, our results apply to the extremal operator

$$
F_{e}\left(D^{2} u, D u, u\right)=\mathcal{M}_{\lambda, \Lambda}^{+}\left(D^{2} u\right)+\gamma|D u|+\delta|u|,
$$

and hence all results concerning maximum principles and existence for the Dirichlet problem apply to operators $H$ satisfying $\left(D_{F_{e}}\right)$, that is, $\left(H_{1}\right)$. In particular, our results give an ABP inequality and existence results for Isaacs operators

$$
\inf _{\beta \in \mathcal{B}} \sup _{\alpha \in \mathcal{A}}\left\{\operatorname{tr}\left(A^{\alpha, \beta}(x) D^{2} u\right)+\vec{b}^{\alpha, \beta}(x) . D u+c^{\alpha, \beta}(x) u\right\}
$$


under a more general hypothesis than $c^{\alpha, \beta}(x) \leq 0$, which was considered in previous works (see for example [CIL], [CKLS] and the references in these papers).

An interesting particular case is obtained by setting $\lambda=\Lambda=1$, in other words, $\mathcal{M}_{\lambda, \Lambda}^{+}\left(D^{2} u\right)=\Delta u$ in $F_{e}$. In a very recent work Hamel, Nadirashvili and Russ considered (among other things) the operator $\Delta u+|D u|$, showed it has two eigenvalues and proved that the domain which minimizes the eigenvalue $\lambda_{1}^{+}(\Delta+|D \cdot|, \Omega)$ on the set of all smooth domains with fixed measure is the ball. Their result depends on the fact that the second-order operator is the Laplacian. It is a very interesting open question to prove that the same holds for more general second-order operators, for example, to prove that $\lambda_{1}^{+}\left(\mathcal{M}_{\lambda, \Lambda}^{+}, \Omega\right)$ is minimized when $\Omega$ is a ball. The analogous question for $\lambda_{1}^{-}$is completely open.

\section{Some known results}

In this section we recall some results for proper uniformly elliptic equations.

We start with the Alexandrov-Bakelman-Pucci estimate for viscosity solutions, see Proposition 3.3 in [CCKS].

Theorem $3.1(\mathrm{ABP})$ Let $f \in L^{N}(\Omega)$. If $u \in C(\bar{\Omega})$ is a $L^{N}$-viscosity solution of

$$
\mathcal{M}_{\lambda, \Lambda}^{+}\left(D^{2} u\right)+\gamma|D u| \geq f \quad \text { in } \quad\{u>0\}
$$

then there exists a constant $B$ depending on $N, \lambda, \Lambda, \gamma$, and $\operatorname{diam}(\Omega)$, such that

$$
\sup _{\Omega} u \leq \sup _{\partial \Omega} u^{+}+B\left\|f^{-}\right\|_{L^{N}(\Omega)} .
$$

Similarly, if $u$ is a $L^{N}$-viscosity solution of $\mathcal{M}_{\lambda, \Lambda}^{-}\left(D^{2} u\right)-\gamma|D u| \leq f$ in $\{u<0\}$, then

$$
\sup _{\Omega} u^{-} \leq \sup _{\partial \Omega} u^{-}+B\left\|f^{+}\right\|_{L^{N}(\Omega)} .
$$

Hence if $F$ satisfies conditions $(S)$ and $(P)$ from the introduction, then $F\left(D^{2} u, D u, u, x\right) \geq f$ implies (3.10), and $F\left(D^{2} u, D u, u, x\right) \leq f$ implies (3.11).

Theorem 3.1 implies a comparison result (Theorem 2.10 in [CCKS]).

Theorem 3.2 Suppose $H$ satisfies $\left(D_{F}\right)$ and $F$ satisfies $\left(H_{1}\right),\left(H_{2}\right)$, and $(P)$. If $u, v \in C(\bar{\Omega})$ are $L^{N}$-viscosity solutions of

$$
\left\{\begin{aligned}
H\left(D^{2} u, D u, u, x\right) & \geq H\left(D^{2} v, D v, v, x\right) & & \text { in } \Omega \\
u & \leq v & & \text { on } \partial \Omega
\end{aligned}\right.
$$


and one of $u, v$ is in $E_{N}$, then $u \leq v$ in $\Omega$.

Now we give a regularity result, which will be needed in the sequel.

Theorem 3.3 Suppose $F$ satisfies $\left(H_{0}\right),\left(H_{1}\right),\left(H_{2}\right)$, and $F(M, p, u, x)$ is convex in $M$. If $u \in C(\bar{\Omega})$ is a viscosity solution of

$$
F\left(D^{2} u, D u, u, x\right)=f \quad \text { in } \quad \Omega
$$

and $f \in L^{p}(\Omega), p \geq N$, then $u \in W_{\mathrm{loc}}^{2, p}(\Omega)$, and for every $\Omega^{\prime} \subset \subset \Omega$

$$
\|u\|_{W^{2, p}\left(\Omega^{\prime}\right)} \leq C\left(\|u\|_{L^{\infty}(\Omega)}+\|f\|_{L^{p}(\Omega)}\right),
$$

where $C$ depends on $\operatorname{dist}\left(\Omega^{\prime}, \partial \Omega\right), \operatorname{diam}(\Omega), p, N, \lambda, \Lambda, \gamma, \delta$.

Proof. In case $F$ is proper (satisfies $(P)$ ), this follows from Theorem 3.1 in [S] (see also the discussion in Sections 8-9 in [CKS]). If $F$ is not proper, by $\left(H_{1}\right)$ the operator $F\left(D^{2} u, D u, u, x\right)-\delta u$ is proper. By applying Theorem 3.1 in $[\mathrm{S}]$ to $F\left(D^{2} u, D u, u, x\right)-\delta u=f-\delta u$ we conclude.

Theorem 3.4 Suppose $F$ satisfies $\left(H_{0}\right),\left(H_{1}\right),\left(H_{2}\right),(P)$, and $F(M, p, u, x)$ is convex in $M$. Then, for any $f \in L^{p}(\Omega), p \geq N$, there exists a unique solution $u \in E_{p}$ of

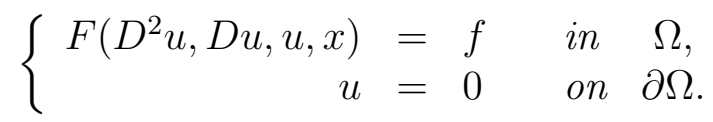

Proof. This is again a consequence of Theorem 3.1 in [S].

Next, we recall the following maximum principle in small domains.

Theorem 3.5 Suppose $H$ satisfies $\left(D_{F}\right)$, and $F$ satisfies $\left(H_{1}\right)$ and $\left(H_{2}\right)$. Then there exists $\varepsilon_{0}>0$, depending on $N, \lambda, \Lambda, \gamma, \delta$, and diam $(\Omega)$, such that if $|\Omega| \leq \varepsilon_{0}$ then for any $u \in C(\bar{\Omega})$

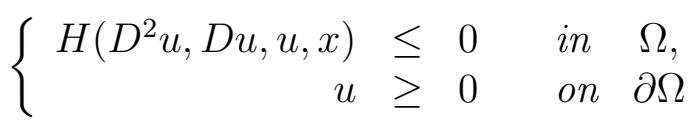

implies $u \geq 0$ in $\Omega$.

Proof. By $\left(H_{1}\right) u$ satisfies

$$
\mathcal{M}_{\lambda, \Lambda}^{-}\left(D^{2} u\right)-\gamma|D u| \leq \delta|u|=\delta u^{-} \quad \text { in } \quad\{u<0\} .
$$

Then we use Theorem 3.1 to conclude, in a standard way.

We shall need the following version of Hopf's boundary lemma. 
Lemma 3.1 Let $\Omega$ be a regular domain and let $u \in E_{N}, u \not \equiv 0$, be $a$ nonnegative solution to

$$
\begin{aligned}
\mathcal{M}_{\lambda, \Lambda}^{-}\left(D^{2} u\right)-\gamma|D u|-\delta u & \leq 0 \quad \text { in } \quad \Omega \\
u & =0 \text { on } \partial \Omega
\end{aligned}
$$

with $\gamma, \delta \in \mathbb{R}$. Then $u>0$ in $\Omega$. Moreover, for each $x_{0} \in \partial \Omega$

$$
\limsup _{x \rightarrow x_{0}} \frac{u\left(x_{0}\right)-u(x)}{\left|x-x_{0}\right|}<0,
$$

where the limit is taken over the set of $x$ for which the angle between $x-x_{0}$ and the outer normal at $x_{0}$ is less than $\pi / 2-\alpha$ for some fixed $\alpha>0$.

Remark. For a general strong maximum principle for degenerate convex elliptic operators, see the paper of M. Bardi, F. Da Lio [BD].

We recall next the following Harnack inequality.

Theorem 3.6 Let $u \in C(\bar{\Omega})$ and $f \in L^{N}(\Omega)$ satisfy $u \geq 0$ in $\Omega$ and

$$
\begin{aligned}
& \mathcal{M}_{\lambda, \Lambda}^{+}\left(D^{2} u\right)+\gamma|D u|+\delta u \geq f \quad \text { in } \quad \Omega, \\
& \mathcal{M}_{\lambda, \Lambda}^{-}\left(D^{2} u\right)-\gamma|D u|-\delta u \leq f \quad \text { in } \quad \Omega .
\end{aligned}
$$

Then for any compact set $K \subset \Omega$

$$
\sup _{K} u \leq C\left\{\inf _{K} u+\|f\|_{L^{N}(\Omega)}\right\}
$$

where $C$ is a constant depending only on $K, \Omega, N, \Lambda, \lambda, \gamma$ and $\delta$.

Proof. First, inequality (3.14) implies the so-called weak Harnack inequality - this was proved by Wang in [W], see also [CC], [CKS] for related results. The fact that inequality (3.13) implies the local maximum principle (see for example [GT], Chapter 9, for the terminology) was proved by Wang in the proper case $(\delta=0)$, and was extended to $\delta>0$ in [BS] - see pages 560-562 of that paper.

\section{Proofs of the main results}

We shall first give an overview of the proofs and list some of the main points in them.

After some preliminary results concerning simplicity and bounds on eventual eigenvalues, we define a class of nonproper operators (in particular those 
with $\widetilde{\lambda}_{1}^{+}>0$, see below), such that one can get comparison results for equations involving these operators through relating them to proper equations and using the results quoted in the previous section. Then the sub- and supersolution method allows us to prove existence and uniqueness results for these equations, which in turn implies, via Krein-Rutman theory, that operators with $\widetilde{\lambda}_{1}^{+}>0$ have two eigenvalues (equal to $\lambda_{1}^{+}, \lambda_{1}^{-}$). The next step is to observe that $\lambda_{1}^{+}, \lambda_{1}^{-}$do not change if one takes only functions $\psi \in E_{p}$ in the definition of $\Psi^{+}, \Psi^{-}$, and then to prove Theorem 1.1, through an approximation procedure in subdomains of $\Omega$. The final quite delicate points are, on one hand, to bridge the gap between $\widetilde{\lambda}_{1}^{+}$and $\lambda_{1}^{+}$, and on the other hand, to show Theorem 1.4 (b) with the right dependence of the constant bounding the $C^{1}$-norm of the supersolution obtained in it. This is accomplished in the key Proposition 4.12, which permits to us to relate our Theorems 1.5-1.10 to corresponding results for proper equations.

\subsection{Some preliminary results}

We start with the trivial proof of Lemma 1.1.

Proof of Lemma 1.1. Suppose $f: \mathbb{R}^{N^{2}+N+1} \rightarrow \mathbb{R}$ is such that $f(t x)=t f(x)$ for all $t \geq 0$. First, $f$ is convex if and only if

$$
f\left(y_{1}\right)-f\left(y_{2}\right) \leq f\left(y_{1}-y_{2}\right) \quad \text { for all } \quad y_{1}, y_{2} \in \mathbb{R}^{N^{2}+N+1} \quad(*) .
$$

Indeed, if $f$ is convex then

$$
f\left(y_{1}\right)=2 f\left(\frac{y_{1}-y_{2}+y_{2}}{2}\right) \leq f\left(y_{1}-y_{2}\right)+f\left(y_{2}\right) .
$$

Whereas if $f$ satisfies $(*)$, then for any $\alpha \in[0,1]$

$$
\begin{aligned}
f\left(\alpha y_{1}+(1-\alpha) y_{2}\right)-\alpha f\left(y_{1}\right) & =f\left(\alpha y_{1}+(1-\alpha) y_{2}\right)-f\left(\alpha y_{1}\right) \\
& \leq f\left((1-\alpha) y_{2}\right)=(1-\alpha) f\left(y_{2}\right),
\end{aligned}
$$

so $f$ is convex.

Define $g(y)=-f(-y)$; we have from $(*)$ that

$$
g\left(y_{1}-y_{2}\right)=-f\left(y_{2}-y_{1}\right) \leq f\left(y_{1}\right)-f\left(y_{2}\right)
$$

and the lemma follows.

Everywhere in the sequel $F$ will denote an operator which satisfies $\left(H_{0}\right)$, $\left(H_{1}\right),\left(H_{2}\right)$, and $\left(D_{F}\right)$.

The following theorem will be used several times. 
Theorem 4.1 Suppose $u, v \in C(\bar{\Omega})$ are viscosity solutions of

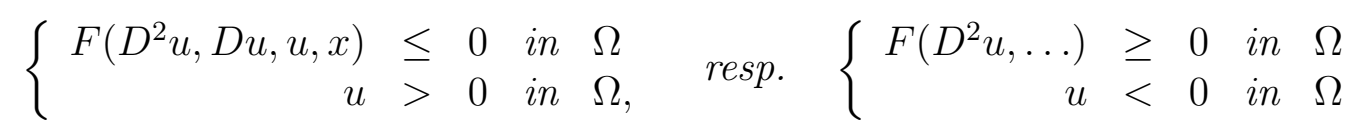

and

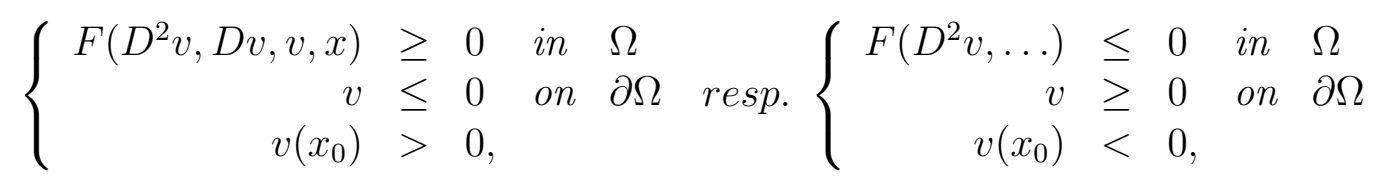

for some point $x_{0} \in \Omega$, where $F$ satisfies $\left(H_{0}\right),\left(H_{1}\right),\left(H_{2}\right)$, and $\left(D_{F}\right)$. Suppose one of $u, v$ is in $E_{N}$. Then $u \equiv t v$ for some $t>0$.

Proof. Let $u, v$ satisfy the first set of inequalities in Theorem 4.1. We suppose first that both $u, v \in E_{N}$. Theorem 4.1 is proved via a variant of a rather typical argument, used for example in the linear setting in [BNV]. Take a compact set $K \subset \Omega$ such that $|\Omega \backslash K| \leq \varepsilon_{0}$, where $\varepsilon_{0}$ is given in Theorem 3.5. Set $z_{t}=v-t u$. If $t$ is large enough $z_{t}<0$ in $K$. By $\left(D_{F}\right)$ and $\left(H_{0}\right)$, we have for all $t \geq 0$

$$
F\left(D^{2} z_{t}, D z_{t}, z_{t}, x\right) \geq F\left(D^{2} v, D v, v, x\right)-t F\left(D^{2} u, D u, u, x\right) \geq 0
$$

in $\Omega$. Since $z_{t} \leq 0$ on $\partial(\Omega \backslash K)$, by using Theorem 3.5 we get $z_{t} \leq 0$ in $\Omega \backslash K$. So, by Hopf's Lemma (Lemma 3.1), either $z_{t} \equiv 0$ in $\Omega$ in which case we are done, or $z_{t}<0$ in $\Omega$. We define

$$
\tau=\inf \left\{t \mid z_{t}<0 \quad \text { in } \Omega\right\} .
$$

Since $v\left(x_{0}\right)>0$ we have $\tau>0$. Now we repeat the same argument for $z_{\tau}$. So, either $z_{\tau} \equiv 0$ in $\Omega$ in which case we are done, or $z_{\tau}<0$ in $\Omega$. In this case there exists $\eta>0$ such that $z_{\tau-\eta}<0$ in $K$. Now we repeat again the same argument for $z_{\tau-\eta}$, which yields a contradiction with the definition of $\tau$.

If the inequalities satisfied by $u, v$ are reversed (second set of inequalities in Theorem 4.1), we consider the function $t u-v$ and use the same argument.

In case one of the functions, say $v$, is only in $C(\bar{\Omega})$, exactly the same argument applies, since (4.15) holds. This is very standard - if $\psi$ is a test function for $z_{t}$, then $\psi+t u$ is a test function for $v$, and vice versa.

Corollary 4.1 If $\mu, \nu \in \mathbb{R}$ and $u, v \in C(\bar{\Omega})$ are such that

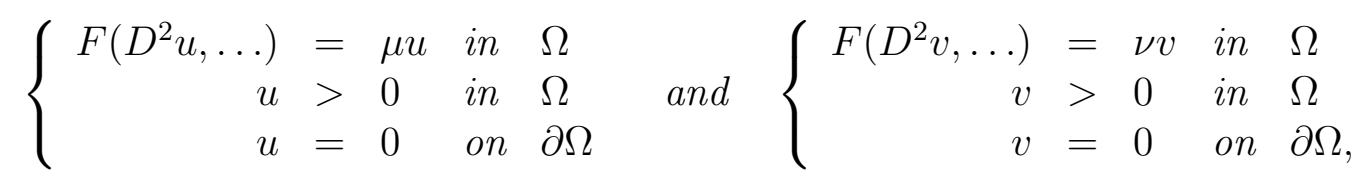

and one of $u, v$ is in $E_{N}$, then $\mu=\nu$ and $u=t v$ for some $t>0$. The same is valid if both functions are negative instead of positive. 
Proof. Suppose $\mu \leq \nu$. Then use Theorem 4.1 with $F\left(D^{2} u, D u, u, x\right)$ replaced by $F\left(D^{2} u, D u, u, x\right)-\mu u$.

Another consequence of Theorem 4.1 is an upper bound of the eigenvalues in terms of the "thickness" of the domain. Before stating it, we recall the following simple fact.

Lemma 4.1 Suppose $u \in C^{2}(B)$ is a radial function, defined on a ball $B$, say $u(x)=g(|x|)$. Then the matrix $D^{2} u(x)$ has $g^{\prime \prime}(|x|)$ as a simple eigenvalue, and $|x|^{-1} g^{\prime}(|x|)$ as an eigenvalue of multiplicity $N-1$.

Proposition 4.1 Suppose $\Omega$ contains a ball $B_{2 R}$, with $R \leq 1$. Then

$$
\lambda_{1}^{+}(F, \Omega) \leq \lambda_{1}^{-}(F, \Omega) \leq \frac{C}{R^{2}}
$$

where $C$ depends on $N, \lambda, \Lambda, \gamma, \delta$.

Proof. Clearly $\lambda_{1}^{-}(\Omega) \leq \lambda_{1}^{-}\left(B_{R}\right)$. We set $\sigma(x)=-\frac{1}{4}\left(|x|^{2}-R^{2}\right)^{2}, \sigma<0$ in $B_{R}$ (this comparison function appears in many other works). Then we have $|D \sigma| \leq|x|\left(R^{2}-|x|^{2}\right)$ and the eigenvalues of $D^{2} \sigma$ are $R^{2}-|x|^{2}$ and $R^{2}-3|x|^{2}$. By $\left(H_{1}\right)$,

$$
F\left(D^{2} \sigma, D \sigma, \sigma, x\right) \leq \mathcal{M}_{\lambda, \Lambda}^{+}\left(D^{2} \sigma\right)+\gamma|D \sigma|+\delta|\sigma|,
$$

so, through an easy computation,

$$
F\left(D^{2} \sigma, D \sigma, \sigma, x\right) \leq-\frac{C_{0}}{R^{2}} \sigma
$$

in $B_{R}$, where $C_{0}$ depends on $N, \lambda, \Lambda, \gamma, \delta$. To get this, we use the previous lemma and the fact that $\mathcal{M}_{\lambda, \Lambda}^{+}(M)=\Lambda \sum_{\left\{e_{i}>0\right\}} e_{i}+\lambda \sum_{\left\{e_{i}<0\right\}} e_{i}$, where $e_{i}$ denote the eigenvalues of $M$.

Suppose now there exists $\lambda \in \mathbb{R}, \psi \in C(\bar{\Omega})$ such that $\psi<0$ in $\Omega$, $F\left(D^{2} \psi, D \psi, \psi, x\right)+\lambda \psi \geq 0$, and $\lambda>\frac{C_{0}}{R^{2}}$. By applying Theorem 4.1 with $F$ replaced by $F\left(D^{2} u, D u, u, x\right)+\frac{C_{0}}{R^{2}} u$ and $\Omega$ replaced by $B_{R}$, we get $\psi=t \sigma$ in $B_{R}$, a contradiction with $\sigma=0, \psi<0$ on $\partial B_{R}$.

Next, for any $\lambda \in \mathbb{R}$ we define the sets

$$
\begin{aligned}
& \widetilde{\Psi}^{+}(F, \Omega, \lambda)=\left\{\psi \mid \psi \in W^{2, p}(\Omega) \quad \forall p<\infty, \quad \inf _{\Omega} \psi=1,\right. \\
& \left.\quad \text { and } F\left(D^{2} \psi, D \psi, \psi, x\right)+\lambda \psi \leq 0 \text { in } \Omega\right\}, \\
& \widetilde{\Psi}^{-}(F, \Omega, \lambda)=\left\{\psi \mid \psi\left(\psi \in W^{2, p}(\Omega) \quad \forall p<\infty, \quad \sup _{\Omega} \psi=-1,\right.\right. \\
& \\
& \left.\quad \text { and } F\left(D^{2} \psi, D \psi, \psi, x\right)+\lambda \psi \geq 0 \text { in } \Omega\right\}
\end{aligned}
$$


(note these sets contain functions which are regular up to $\partial \Omega$ ), and the following quantities

$$
\begin{aligned}
& \widetilde{\lambda}_{1}^{+}(F, \Omega)=\sup \left\{\lambda \mid \widetilde{\Psi}^{+}(F, \Omega, \lambda) \neq \emptyset\right\}, \\
& \widetilde{\lambda}_{1}^{-}(F, \Omega)=\sup \left\{\lambda \mid \widetilde{\Psi}^{-}(F, \Omega, \lambda) \neq \emptyset\right\} .
\end{aligned}
$$

By using these definitions and by setting $\psi \equiv 1$ in (4.16), we obtain the following bounds.

Proposition 4.2 We have $\widetilde{\lambda}_{1}^{-} \leq \lambda_{1}^{-}$and

$$
-\delta \leq-\|c\|_{\infty} \leq \widetilde{\lambda}_{1}^{+} \leq \lambda_{1}^{+} \leq \lambda_{1}^{-} \leq \frac{C}{R^{2}}
$$

where $c(x)=F(0,0,1, x)$, and $C$ is the constant from Proposition 4.1.

\subsection{First comparison and existence results for non- proper equations}

We are now going to show that a version of the ABP inequality (and hence the maximum principle) holds for operators with positive $\widetilde{\lambda}_{1}^{+}, \widetilde{\lambda}_{1}^{-}$.

Proposition 4.3 Suppose F satisfies $\left(H_{0}\right),\left(H_{1}\right),\left(H_{2}\right),\left(D_{F}\right)$. Suppose there exists a function $\psi_{1} \in \widetilde{\Psi}^{+}(F, \Omega, 0)$. Then for any $u \in C(\bar{\Omega}), f \in L^{N}(\Omega)$ the inequality $F\left(D^{2} u, D u, u, x\right) \geq f$ in $\Omega$ implies

$$
\sup _{\Omega} u \leq C\left(\sup _{\partial \Omega} u^{+}+\left\|f^{-}\right\|_{L^{N}(\Omega)}\right)
$$

where $C$ depends on $\operatorname{diam}(\Omega), \lambda, \Lambda, \gamma, \delta$, and $\left\|\psi_{1}\right\|_{C^{1}(\Omega)}$. Similarly, if there exists a function $\psi_{2} \in \widetilde{\Psi}^{-}(F, \Omega, 0)$ then the inequality $F\left(D^{2} u, D u, u, x\right) \leq f$ implies

$$
\sup _{\Omega} u^{-} \leq C\left(\sup _{\partial \Omega} u^{-}+\left\|f^{+}\right\|_{L^{N}(\Omega)}\right) .
$$

Note that $\widetilde{\lambda}_{1}^{+}>0$ (resp. $\left.\widetilde{\lambda}_{1}^{-}>0\right)$ implies the existence of $\psi_{1} \in \widetilde{\Psi}^{+}(F, \Omega, 0)$ (resp. $\psi_{2} \in \widetilde{\Psi}^{-}(F, \Omega, 0)$ ).

Proof. The proof is symmetric for both cases, so we present it only in the case when there exists a function $\phi \in \widetilde{\Psi}^{+}(F, \Omega, 0)$. Set $A=\|\phi\|_{C^{1}(\Omega)}$ (recall $W^{2, p}(\Omega)$ embeds into $\left.C^{1, \alpha}(\Omega)\right)$.

We define the following operator

$$
F_{\phi}(M, p, v, x)=F\left(\phi M+p \otimes D \phi+v D^{2} \phi, \phi p+v D \phi, v \phi, x\right)
$$


(here and in the sequel $p \otimes q$ denotes the matrix $\left(p_{i} q_{j}\right)_{i j}$, for $\left.p, q \in \mathbb{R}^{N}\right)$.

Claim. Hypotheses $(S)$ and $(P)$ from the introduction are satisfied by $F_{\phi}$ (so Theorem 3.1 applies to $\left.F_{\phi}\right)$.

Before continuing, we recall the following properties of Pucci operators.

Lemma 4.2 Let $M, N \in \mathcal{S}_{N}, p, q \in \mathbb{R}^{N}$, and $\phi(x) \in C(\bar{\Omega})$ be such that $0<a \leq \phi(x) \leq A$. Then

$$
\begin{aligned}
\mathcal{M}_{\lambda, \Lambda}^{-}(M)+ & \mathcal{M}_{\lambda, \Lambda}^{-}(N) \leq \mathcal{M}_{\lambda, \Lambda}^{-}(M+N) \leq \mathcal{M}_{\lambda, \Lambda}^{-}(M)+\mathcal{M}_{\lambda, \Lambda}^{+}(N) \\
& \mathcal{M}_{\lambda a, \Lambda A}^{-}(M) \leq \mathcal{M}_{\lambda, \Lambda}^{-}(\phi M) \leq \mathcal{M}_{\lambda A, \Lambda a}^{-}(M) \\
& -C(\lambda, \Lambda, N)|p||q| \leq \mathcal{M}_{\lambda, \Lambda}^{-}(p \otimes q) \leq \Lambda|p||q|
\end{aligned}
$$

Proof. For the first two lines, simply use the definition of $\mathcal{M}_{\lambda, \Lambda}^{-}$. For the third line, note that for any $y \in \mathbb{R}^{N}$ we have

$$
<(p \otimes q) y, y>=<p, y><q, y>\leq\left.|p||q \|| y\right|^{2},
$$

so $\widetilde{M}=p \otimes q-|p||q| I$ is negative definite and $\mathcal{M}_{\lambda, \Lambda}^{-}(\widetilde{M})=\Lambda \operatorname{tr}(\widetilde{M})$. Of course $|\operatorname{tr}(p \otimes q)| \leq|p||q|$. So, by what we already know,

$$
\mathcal{M}_{\lambda, \Lambda}^{-}(\widetilde{M})+\mathcal{M}_{\lambda, \Lambda}^{-}(|p||q| I) \leq \mathcal{M}_{\lambda, \Lambda}^{-}(p \otimes q) \leq \mathcal{M}_{\lambda, \Lambda}^{-}(\widetilde{M})+\mathcal{M}_{\lambda, \Lambda}^{+}(|p||q| I),
$$

and the lemma follows, with $C(\lambda, \Lambda, N)=\Lambda(N+1)-\lambda N$.

We continue with the proof of Proposition 4.3. Let $M, N \in \mathcal{S}_{N}$, and $p, q \in \mathbb{R}^{N}$. Then by $\left(H_{1}\right)$ and the above lemma

$$
\begin{aligned}
F_{\phi}(M, p, v, x)-F_{\phi}(N, q, v, x) \geq & \mathcal{M}_{\lambda, \Lambda}^{-}(\phi(M-N)+D \phi \otimes(p-q)) \\
& -\gamma|p-q| \\
\geq & \mathcal{M}_{\lambda, \Lambda}^{-}(\phi(M-N)) \\
& +\mathcal{M}_{\lambda, \Lambda}^{-}(D \phi \otimes(p-q))-\gamma|p-q| \\
\geq & \mathcal{M}_{\lambda, \Lambda A}^{-}(M-N)-(C A+\gamma)|p-q|,
\end{aligned}
$$

where $C=C(\lambda, \Lambda, N)$ is the constant from Lemma 4.2. In the same way we obtain

$$
F_{\phi}(M, p, v, x)-F_{\phi}(N, q, v, x) \leq \mathcal{M}_{\lambda, \Lambda A}^{+}(M-N)+(C A+\gamma)|p-q|
$$

(recall $\left.\mathcal{M}_{\lambda, \Lambda}^{-}(M)=-\mathcal{M}_{\lambda, \Lambda}^{+}(-M)\right)$. So $F_{\phi}$ satisfies $(S)$, with modified constants. Let us prove now that $F_{\phi}$ satisfies $(P)$. Let $v_{1}, v_{2} \in \mathbb{R}$ be such that 
$v=v_{1}-v_{2} \geq 0$. Then by the definition of $F_{\phi},\left(D_{F}\right),\left(H_{0}\right)$ and $\phi \in \widetilde{\Psi}^{+}(F, \Omega, 0)$ we get

$$
\begin{aligned}
F_{\phi}\left(M, p, v_{1}, x\right)-F_{\phi}\left(M, p, v_{2}, x\right) & \leq F\left(v D^{2} \phi, v D \phi, v \phi, x\right) \\
& =v F\left(D^{2} \phi, D \phi, \phi, x\right) \leq 0 .
\end{aligned}
$$

So $F_{\phi}(M, p, v, x)$ is nonincreasing in $v$ and the claim follows.

Now set $v=\frac{u}{\phi}$. Then by using that $F\left(D^{2} u, D u, u, x\right) \geq f$ we easily obtain $F_{\phi}\left(D^{2} v, D v, v, x\right) \geq f$. Now we use Theorem 3.1 for $F_{\phi}$, which yields

$$
\sup _{\Omega} v \leq \sup _{\partial \Omega} v^{+}+C\left\|f^{-}\right\|_{L^{N}(\Omega)}
$$

where $C$ depends on the appropriate quantities. Note that $A^{-1} u \leq v \leq u$. Proposition 4.3 follows.

The following comparison result is an immediate consequence of $\left(D_{F}\right)$ and Proposition 4.3.

Proposition 4.4 Suppose $\widetilde{\lambda}_{1}^{+}(F)>0$. If $u, v \in C(\bar{\Omega})$ are such that

$$
\left\{\begin{aligned}
F\left(D^{2} u, D u, u, x\right) & \geq F\left(D^{2} v, D v, v, x\right) & & \text { in } \quad \Omega, \\
u & \leq v & & \text { on } \partial \Omega,
\end{aligned}\right.
$$

and one of $u, v$ is in $E_{N}$, then $u \leq v$ in $\Omega$.

Proof. Recall $\widetilde{\lambda}_{1}^{+}(F)>0$ implies that $\widetilde{\Psi}^{+}(F, \Omega, 0)$ is not empty. Then we conclude with the help of $\left(D_{F}\right)$ and Proposition 4.3, applied to $v-u$.

Remark. Note that $\widetilde{\lambda}_{1}^{-}(F)>0$ does not imply comparison.

Next, we prove that $\widetilde{\lambda}_{1}^{+}>0$ is sufficient for the solvability of the Dirichlet problem.

Proposition 4.5 Suppose $F$ satisfies hypotheses $\left(H_{0}\right),\left(H_{1}\right),\left(H_{2}\right)$, and $\left(D_{F}\right)$. If $\tilde{\lambda}_{1}^{+}(F)>0$ then for any $f \in L^{p}(\Omega), p \geq N$, there exists a unique viscosity solution $u \in E_{p}$ of

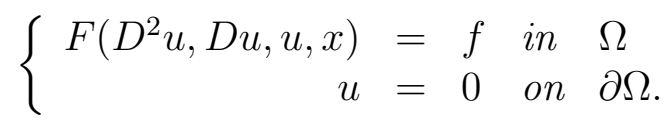

In addition $f \geq 0$ in $\Omega$ implies $u \leq 0$ in $\Omega$, and $f \leq 0$ implies $u \geq 0$.

Proof. Suppose first $f \in C(\bar{\Omega})$. By $\widetilde{\lambda}_{1}^{+}>0$ and the definition of $\widetilde{\lambda}_{1}^{+}$, there exists a function $\psi_{1} \in \widetilde{\Psi}^{+}\left(F, \Omega, \frac{1}{2} \widetilde{\lambda}_{1}^{+}\right)$, that is, $\psi_{1} \geq 1$ in $\Omega$ and

$$
F\left(D^{2}\left(k \psi_{1}\right), D\left(k \psi_{1}\right), k \psi_{1}, x\right) \leq-\frac{\widetilde{\lambda}_{1}^{+}}{2} k \psi_{1} \quad \text { for all } k>0
$$


(in this inequality we have used the fact that $F$ is homogeneous). We fix $k_{1}$ such that $\tilde{\lambda}_{1}^{+} k_{1} \geq 2\|f\|_{L^{\infty}}$. Then the positive function $k_{1} \psi_{1}$ is a supersolution of the equation $F\left(D^{2} u, D u, u, x\right)=f$.

Similarly, since $\widetilde{\lambda}_{1}^{-} \geq \widetilde{\lambda}_{1}^{+}>0$ we can find a function $\psi_{2} \in \widetilde{\Psi}^{-}\left(F, \Omega, \frac{1}{2} \widetilde{\lambda}_{1}^{-}\right)$, such that $\psi_{2} \leq-1$ in $\Omega$ and

$$
F\left(D^{2}\left(k \psi_{2}\right), D\left(k \psi_{2}\right), k \psi_{2}, x\right) \geq-\frac{\widetilde{\lambda}_{1}^{-}}{2} k \psi_{2} \quad \text { for all } k>0 .
$$

Fixing $k_{2}$ such that $\tilde{\lambda}_{1}^{-} k_{2} \geq 2\|f\|_{L^{\infty}}$, the negative function $k_{2} \psi_{2}$ is a subsolution of the equation $F\left(D^{2} u, D u, u, x\right)=f$.

Now we use the following (standard) lemma.

Lemma 4.3 Under the hypotheses of Proposition 4.5, suppose $u_{0} \in E_{p}$ is a subsolution and $v_{0} \in E_{p}$ is a supersolution of $F\left(D^{2} u, D u, u, x\right)=f$, where $f \in C(\bar{\Omega})$. Suppose in addition that $u_{0} \leq v_{0}$ in $\Omega, u_{0} \leq 0$ on $\partial \Omega$, and $v_{0} \geq 0$ on $\partial \Omega$. Then there exists a solution $u \in E_{p}$ of

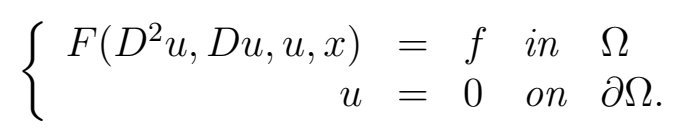

Proof. It is clear that hypothesis $\left(H_{1}\right)$ implies that $F(M, p, u, x)-\delta u$ is nonincreasing in $u$. Therefore, by Theorem 3.4 we can solve the hierarchy of problems

$$
\left\{\begin{aligned}
F\left(D^{2} u_{n+1}, D u_{n+1}, u_{n+1}, x\right)-\delta u_{n+1} & =f-\delta u_{n} & & \text { in } \Omega \\
u_{n+1} & =0 & & \text { on } \partial \Omega .
\end{aligned}\right.
$$

By Theorem 3.2 we have $u_{0} \leq u_{1} \leq \ldots \leq v_{0}$. Hence $u_{n}$ tends pointwise to a function $u$, and by interior estimates (Theorem 3.3) in $W_{\text {loc }}^{2, p}$, hence locally uniformly. Then by the viscosity solutions theory (see for example Theorem 3.8 in [CCKS] $) u$ is a solution of $F\left(D^{2} u, D u, u, x\right)=f$ in $\Omega$. By Theorem 3.2 we have $w_{1} \leq u_{n} \leq w_{2}$ for all $n$, where $w_{1}, w_{2} \in E_{p}$ are the solutions of

$$
\left\{\begin{aligned}
F\left(D^{2} w_{i}, D w_{i}, w_{i}, x\right)-\delta w_{i} & =\varepsilon_{i} L & & \text { in } \Omega \\
w_{i} & =0 & \text { on } & \partial \Omega
\end{aligned}\right.
$$

where $\varepsilon_{1}=1, \varepsilon_{2}=-1, L=\sup _{n}\left\|f-\delta u_{n}\right\|_{L^{\infty}(\Omega)}\left(L\right.$ depends on $\left.f, \delta, u_{0}, v_{0}\right)$. Hence $u \in C(\bar{\Omega})$ and $u=0$ on $\partial \Omega$.

To finish the proof of Proposition 4.5, if $f \in L^{p}$ is not in $C(\bar{\Omega})$, we take a sequence $f_{n} \in C(\bar{\Omega})$ which tends to $f$ in the $L^{p}$-norm. By what we already proved, there exists a solution $u_{n} \in E_{p}$ of $F\left(D^{2} u_{n}, D u_{n}, u_{n}, x\right)=f_{n}$. Then by $\left(D_{F}\right)$ we obtain

$$
F\left(D^{2}\left(u_{n}-u_{m}\right), D\left(u_{n}-u_{m}\right), u_{n}-u_{m}, x\right) \geq f_{n}-f_{m},
$$




$$
F\left(D^{2}\left(u_{m}-u_{n}\right), D\left(u_{m}-u_{n}\right), u_{m}-u_{n}, x\right) \geq f_{m}-f_{n},
$$

for any $m, n$. By the ABP inequality (Proposition 4.3), which we already proved,

$$
\left\|u_{m}-u_{n}\right\|_{L^{\infty}(\Omega)} \leq C\left\|f_{m}-f_{n}\right\|_{L^{N}(\Omega)},
$$

hence $\left\{u_{n}\right\}$ is a Cauchy sequence in $C(\bar{\Omega})$ and tends uniformly to a function $u \in C(\bar{\Omega})$. We conclude by passing to the limit again. The function $u$ is in $E_{p}$, by Theorem 3.3 .

The uniqueness and the final statement of Proposition 4.5 follow from Proposition 4.4.

\subsection{On existence of eigenvalues. Proof of Theorems 1.1 and 1.2}

We are now able to prove the existence of two principal eigenvalues and eigenfunctions, when $\widetilde{\lambda}_{1}^{+}>0$.

Proposition 4.6 Suppose $\widetilde{\lambda}_{1}^{+}>0$. Then there exist positive numbers $\Lambda_{1}^{+}, \Lambda_{1}^{-}$, and functions $\varphi_{1}^{+}, \varphi_{1}^{-} \in E_{p}$ for all $p<\infty$, such that

$$
\left\{\begin{aligned}
F\left(D^{2} \varphi_{1}^{+}, D \varphi_{1}^{+}, \varphi_{1}^{+}, x\right) & =-\Lambda_{1}^{+} \varphi_{1}^{+}, & \varphi_{1}^{+}>0 & \text { in } \Omega \\
\varphi_{1}^{+} & =0 & & \text { on } \partial \Omega
\end{aligned}\right.
$$

and

$$
\left\{\begin{aligned}
F\left(D^{2} \varphi_{1}^{-}, D \varphi_{1}^{-}, \varphi_{1}^{-}, x\right) & = & -\Lambda_{1}^{-} \varphi_{1}^{-}, & \varphi_{1}^{-}<0 & \text { in } & \Omega \\
\varphi_{1}^{-} & =0 & & & \text { on } & \partial \Omega
\end{aligned}\right.
$$

Proof. Recall $F$ is convex and positively homogeneous. We have proved (Propositions 4.4 and 4.5) that $(C P)$ holds for $F$ and the Dirichlet problem is uniquely solvable for $F$. Moreover the Hopf lemma (Lemma 3.1) holds for $F$. Then we can use an adaptation of the classical Krein-Rutman Theorem for a convex operator in a cone to establish the existence of $\Lambda_{1}^{+}>0$ and $\varphi_{1}^{+}$. The argument is carried out in $[\mathrm{Q}]$ or $[\mathrm{FQ}]$. Since we can use exactly the same argument, we refer to these papers for details.

The same argument can be used for $G$, this yields the existence of $\Lambda_{1}^{-}>0$ and $\varphi_{1}^{-}$.

Proposition 4.7 If $\widetilde{\lambda}_{1}^{+}>0$ and $\Lambda_{1}^{+}, \Lambda_{1}^{-}$are as in the previous proposition then $\Lambda_{1}^{+}=\lambda_{1}^{+}$and $\Lambda_{1}^{-}=\lambda_{1}^{-}$. 
Proof. From Proposition 4.6 (the existence of $\varphi_{1}^{+}$) and the definition of $\lambda_{1}^{+}$ we have $\Lambda_{1}^{+} \leq \lambda_{1}^{+}$.

Suppose there exists $\varepsilon>0$ such that $\Lambda_{1}^{+}<\lambda_{1}^{+}-\varepsilon$. By the definition of $\lambda_{1}^{+}$we can take $\phi \in C(\bar{\Omega})$ such that

$$
F\left(D^{2} \phi, D \phi, \phi, x\right)+\left(\lambda_{1}^{+}-\varepsilon\right) \phi \leq 0, \quad \phi>0 \quad \text { in } \quad \Omega .
$$

Since

$F\left(D^{2} \varphi_{1}^{+}, D \varphi_{1}^{+}, \varphi_{1}^{+}, x\right)+\left(\lambda_{1}^{+}-\varepsilon\right) \varphi_{1}^{+}>F\left(D^{2} \varphi_{1}^{+}, D \varphi_{1}^{+}, \varphi_{1}^{+}, x\right)+\Lambda_{1}^{+} \varphi_{1}^{+}=0$ in $\Omega$,

and $\varphi_{1}^{+}>0$ in $\Omega, \varphi_{1}^{+}=0$ on $\partial \Omega$, Theorem 4.1 implies that $\phi=t \varphi_{1}^{+}$for some $t>0$, which is a contradiction. By replacing $F$ by $G$ in this argument we get $\Lambda_{1}^{-}=\lambda_{1}^{-}$.

\section{Lemma 4.4 Set}

$$
\begin{array}{ll}
\Psi_{0}^{+}(F, \Omega, \lambda)=\left\{\psi \in E_{p}, \quad \forall p<\infty \quad\right. & F\left(D^{2} \psi, D \psi, \psi, x\right)+\lambda \psi \leq 0 \text { in } \Omega \\
& \text { and } \psi>0 \text { in } \Omega\} \\
& \\
\Psi_{0}^{-}(F, \Omega, \lambda)=\left\{\psi \in E_{p}, \quad \forall p<\infty \quad\right. & F\left(D^{2} \psi, D \psi, \psi, x\right)+\lambda \psi \geq 0 \text { in } \Omega \\
& \text { and } \psi<0 \text { in } \Omega\} .
\end{array}
$$

Then

$$
\begin{aligned}
& \lambda_{1}^{+}(F, \Omega)=\bar{\lambda}_{1}^{+}(F, \Omega):=\sup \left\{\lambda \mid \Psi_{0}^{+}(F, \Omega, \lambda) \neq \emptyset\right\}, \\
& \lambda_{1}^{-}(F, \Omega)=\bar{\lambda}_{1}^{-}(F, \Omega):=\sup \left\{\lambda \mid \Psi_{0}^{-}(F, \Omega, \lambda) \neq \emptyset\right\} .
\end{aligned}
$$

Proof. If $\widetilde{\lambda}_{1}^{+}>0$ this follows from the previous proposition, since clearly $\Lambda_{1}^{+}(F, \Omega) \leq \bar{\lambda}_{1}^{+}(F, \Omega) \leq \lambda_{1}^{+}(F, \Omega)$ (from Proposition 4.6 and the definitions of $\left.\lambda_{1}^{+}, \bar{\lambda}_{1}^{+}\right)$.

Note that if $F$ is a proper operator then $\widetilde{\lambda}_{1}^{+}(F) \geq 0$ (since $\psi \equiv 1$ is in $\left.\widetilde{\Psi}^{+}(F, \Omega, 0)\right)$. On the other hand $F\left(D^{2} u, D u, u, x\right)-\delta u$ is nonincreasing in $u$. Then, setting $\widehat{F}\left(D^{2} u, D u, u, x\right)=F\left(D^{2} u, D u, u, x\right)-(\delta+1) u$ we have $\widetilde{\lambda}_{1}^{+}(\widehat{F}) \geq 1$, so, by what we already proved,

$$
\lambda_{1}^{+}(F)+\delta+1=\lambda_{1}^{+}(\widehat{F})=\bar{\lambda}_{1}^{+}(\widehat{F})=\bar{\lambda}_{1}^{+}(F)+\delta+1 .
$$

Theorem 4.2 Suppose $F$ satisfies hypotheses $\left(H_{0}\right),\left(H_{1}\right),\left(H_{2}\right)$, and $\left(D_{F}\right)$. If $\lambda_{1}^{+}(F, \Omega)>0$ then there exist functions $\varphi_{1}^{+}, \varphi_{1}^{-} \in E_{p}$ for all $p<\infty$, such that $\varphi_{1}^{+}>0, \varphi_{1}^{-}<0$ in $\Omega, \varphi_{1}^{+}=0, \varphi_{1}^{-}=0$ on $\partial \Omega$, and

$$
F\left(D^{2} \varphi_{1}^{+}, D \varphi_{1}^{+}, \varphi_{1}^{+}, x\right)=-\lambda_{1}^{+} \varphi_{1}^{+}, \quad F\left(D^{2} \varphi_{1}^{-}, D \varphi_{1}^{-}, \varphi_{1}^{-}, x\right)=-\lambda_{1}^{-} \varphi_{1}^{-} .
$$


Proof. Take a sequence of smooth domains $\Omega_{n} \subset \subset \Omega$, such that $\Omega_{n} \rightarrow \Omega$ as $n \rightarrow \infty$. By the previous lemma $\lambda_{1}^{+}(\Omega)>0$ implies $\widetilde{\lambda}_{1}^{+}\left(\Omega_{n}\right)>0$, for each $n$. By Propositions 4.6 and 4.7 there exist functions $\varphi_{1}^{(n)} \in W_{\text {loc }}^{2, p}\left(\Omega_{n}\right) \cap C\left(\overline{\Omega_{n}}\right)$, $\forall p<\infty$, such that $\varphi_{1}^{(n)}>0$ in $\Omega_{n}, \varphi_{1}^{(n)}=0$ on $\partial \Omega_{n}$, and

$$
F\left(D^{2} \varphi_{1}^{(n)}, D \varphi_{1}^{(n)}, \varphi_{1}^{(n)}, x\right)=-\lambda_{1}^{(n)} \varphi_{1}^{(n)} \quad \text { in } \Omega_{n} .
$$

where $\lambda_{1}^{(n)}=\lambda_{1}^{+}\left(\Omega_{n}\right)$. Likewise, there exist negative eigenfunctions in $\Omega_{n}$ corresponding to $\lambda_{1}^{-}\left(\Omega_{n}\right)$.

Clearly $\left\{\lambda_{1}^{(n)}\right\}$ is nonincreasing and bounded below by $\lambda_{1}^{+}(\Omega)$. Hence $\left\{\lambda_{1}^{(n)}\right\}$ converges to a number $\Lambda_{1}$, with $\Lambda_{1} \geq \lambda_{1}^{+}(\Omega)>0$. Proposition 4.2 provides an upper bound for $\Lambda_{1}$.

The argument which follows is inspired by [BNV]. Fix a point $x_{0} \in \Omega$ and renormalize $\varphi_{1}^{(n)}$ so that $\varphi_{1}^{(n)}\left(x_{0}\right)=1$ (of course $k \varphi_{1}^{(n)}$ satisfies the same equation as $\varphi_{1}^{(n)}$, for any $\left.k>0\right)$. Fix a compact set $K \subset \Omega$ such that $x_{0} \in K$ and $|\Omega \backslash K|<\beta$, where $\beta$ is to be chosen later.

By the Harnack inequality (Theorem 3.6) we have for large $n$

$$
\left\|\varphi_{1}^{(n)}\right\|_{L^{\infty}(K)} \leq C_{1} \varphi_{1}^{(n)}\left(x_{0}\right)=C_{1}
$$

By the hypotheses on $F$ - the homogeneity, $\left(H_{1}\right)$, and the fact that

$$
F(M, p, u, x) \leq F(M, p, 0, x)+F(0,0, u, x),
$$

we get

$$
\begin{aligned}
F\left(D^{2}\left(\varphi_{1}^{(n)}-C_{1}\right), D\left(\varphi_{1}^{(n)}-C_{1}\right), 0, x\right) \geq \quad & F\left(D^{2}\left(\varphi_{1}^{(n)}-C_{1}\right), \ldots\right) \\
& -F\left(0,0, \varphi_{1}^{(n)}-C_{1}, x\right) \\
\geq \quad & F\left(D^{2} \varphi_{1}^{(n)}, \ldots\right)-C_{1} F(0,0,1, x) \\
& -\delta\left|\varphi_{1}^{(n)}-C_{1}\right| \\
\geq & \left(-\lambda_{1}^{(n)}-\delta\right) \varphi_{1}^{(n)}-2 C_{1} \delta
\end{aligned}
$$

(we have written $F\left(D^{2} u, \ldots\right.$ ) for $F\left(D^{2} u, D u, u, x\right)$ ). By applying the ABP inequality (Theorem 3.1) in the domain $\Omega_{n} \backslash K$ we get

$$
\sup _{\Omega_{n} \backslash K}\left(\varphi_{1}^{(n)}-C_{1}\right) \leq(1 / 2) \sup _{\Omega_{n} \backslash K} \varphi_{1}^{(n)}+C_{2},
$$

provided we choose $\beta=\left(2 B\left(\Lambda_{1}+\delta+1\right)\right)^{-N}$, where $B$ is the constant from Theorem 3.1. Hence

$$
\left\|\varphi_{1}^{(n)}\right\|_{L^{\infty}(\Omega)} \leq 2\left(C_{1}+C_{2}\right) .
$$


In addition, we proved that

$$
F\left(D^{2} \varphi_{1}^{(n)}, D \varphi_{1}^{(n)}, 0, x\right) \geq-\left(\Lambda_{1}+\delta+1+2 C_{1} \delta\right)=:-C_{3}
$$

in $\Omega_{n}$. Therefore, by Theorem 3.2,

$$
\varphi_{1}^{(n)} \leq C_{3} w_{0}
$$

in $\Omega_{n}$, where $w_{0} \in E_{p}, p<\infty$, is such that

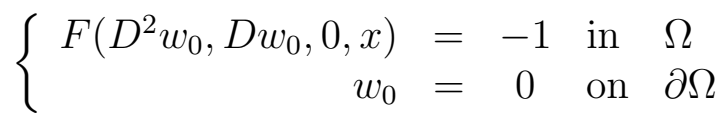

(this problem is solvable, see Theorem 3.4).

Now, by interior $W^{2, p}$-estimates, the sequence $\varphi_{1}^{(n)}$ converges in $W_{\text {loc }}^{2, p}$ to a nonnegative function $\varphi_{1}^{+}$, which is bounded in $\Omega, \varphi_{1}^{+}\left(x_{0}\right)=1$, and

$$
F\left(D^{2} \varphi_{1}^{+}, D \varphi_{1}^{+}, \varphi_{1}^{+}, x\right)=-\Lambda_{1} \varphi_{1}^{+}
$$

in $\Omega$. By the strong maximum principle $\varphi_{1}^{+}>0$ in $\Omega$. By (4.18) $\varphi_{1}^{+} \leq C_{3} w_{0}$ in $\Omega$ - so in particular $\varphi_{1}^{+} \in C(\bar{\Omega})$ and $\varphi_{1}^{+}=0$ on $\partial \Omega$.

Recall that $\Lambda_{1} \geq \lambda_{1}^{+}$. On the other hand the definition of $\lambda_{1}^{+}$and (4.19) imply $\Lambda_{1} \leq \lambda_{1}^{+}$. Exactly the same argument (reversing signs and inequalities where appropriate) can be carried out for $\lambda_{1}^{(n)}=\lambda_{1}^{-}\left(\Omega_{n}\right)$ and $\varphi_{1}^{(n)}<0$ in $\Omega$ (then $G\left(D^{2}\left(-\varphi_{1}^{(n)}\right), \ldots\right)=-\lambda_{1}^{-}\left(-\varphi_{1}^{(n)}\right)$ in $\Omega$ ). Theorem 4.2 is proved.

Proof of Theorem 1.1 We have just proved that operators with positive $\lambda_{1}^{+}$have a principal eigenvalue (equal to $\lambda_{1}^{+}$). So, to show that any $F$ has two principal eigenvalues and eigenfunctions, it is enough to consider the operator $F(M, p, u, x)-\left(\lambda_{1}^{+}(F)+1\right) u$, whose $\lambda_{1}^{+}$is equal to 1 .

The upper bound on $\varphi_{1}^{+}, \varphi_{1}^{-}$follows from the proof of Theorem 4.2. The fact that no other eigenvalue can correspond to a positive (negative) eigenfunction is a consequence of Corollary 4.1. Theorem 1.1 is proved.

The following lower bound on $\lambda_{1}^{+}$in terms of the measure of the domain is an easy consequence of Theorem 1.1 and Theorem 3.1.

Proposition 4.8 Under the hypotheses of Theorem 1.1

$$
\lambda_{1}^{+}(F, \Omega) \geq \frac{1}{B|\Omega|^{\frac{1}{N}}}-\delta
$$

where $B$ is the constant from Theorem 3.1. 
Proof. Recall $F\left(D^{2} u, D u, u, x\right)-\delta u$ satisfies the hypotheses of Theorem 3.1 and apply this theorem to

$$
F\left(D^{2} \varphi_{1}^{+}, D \varphi_{1}^{+}, \varphi_{1}^{+}, x\right)-\delta \varphi_{1}^{+}=-\left(\lambda_{1}^{+}+\delta\right) \varphi_{1}^{+} .
$$

Proof of Theorem 1.2 We shall use Theorem 4.1 (the first set of inequalities), with $F\left(D^{2} u, D u, u, x\right)$ replaced by $F\left(D^{2} u, D u, u, x\right)+\lambda_{1}^{+} u$.

Suppose $u_{1}=u$ satisfies (1.3). Then we apply Theorem 4.1 with $u=\varphi_{1}^{+}$ and $v=u_{1}$.

If $u_{1}=u$ satisfies (1.2) then either $u_{1}$ is positive somewhere, so $u_{1}$ satisfies (1.3) and we are in the previous case, or $u_{1}$ is a negative eigenfunction, so $\lambda_{1}^{+}=\lambda_{1}^{-}$, by Theorem 1.1. Then we apply Theorem 4.1 with $u=\varphi_{1}^{+}$and $v=-u_{1}$. Theorem 1.2 is proved.

\subsection{Proof of $\lambda_{1}^{+}=\widetilde{\lambda}_{1}^{+}$. Proof of Theorem 1.4}

In the sequel we shall need the following boundary Lipschitz estimate for fully nonlinear equations. It is simple and probably known, yet we have not found a reference, so for the reader's convenience we shall include the proof. For the case of a linear equation, see Problem 3.6 in [GT].

Proposition 4.9 Suppose $F$ satisfies $\left(H_{1}\right)$ and $\Omega$ satisfies an uniform exterior sphere condition. Suppose $u \in C(\bar{\Omega})$ satisfies $F\left(D^{2} u, D u, u, x\right)=f$, $u=0$ on $\partial \Omega$, where $f \in L^{\infty}(\Omega)$. Then there exists a constant $k$ depending on $N, \lambda, \Lambda, \gamma, \delta$, $\operatorname{diam}(\Omega),\|u\|_{L^{\infty}(\Omega)},\|f\|_{L^{\infty}(\Omega)}$, and the radius of the exterior spheres, such that for each $x_{0} \in \partial \Omega$

$$
|u(x)| \leq k\left|x-x_{0}\right| \quad \text { for each } x \in \Omega .
$$

Proof. We use the barrier $w(x)=l\left(R^{-p}-|x-y|^{-p}\right)$ for sufficiently large $p$ and $l$. Here $y$ is the center of the exterior ball touching $\partial \Omega$ at $x_{0}$ and $R$ is its radius. We replace $F$ by $F-\delta u$ (so that $F-\delta u$ be proper) and $f$ by $f-\delta u$. Since $w$ is radial, with the aid of Lemma 4.1 it is simple to see that

$$
\mathcal{M}_{\lambda, \Lambda}^{+}\left(D^{2} w\right)+b|D w| \leq-l
$$

provided $p$ is taken large enough. We then fix $l \geq\|f-\delta u\|_{\infty}$. Hence

$$
\begin{aligned}
F\left(D^{2} w, D w, w, x\right)-\delta w & \leq F\left(D^{2} u, D u, u, x\right)-\delta u \quad \text { in } \Omega \\
w & \geq u \text { on } \partial \Omega,
\end{aligned}
$$

so the comparison between $u$ and $w$ permits to conclude.

Next, we prove that the principal eigenvalues are continuous with respect to the domain. 
Proposition 4.10 Suppose $\Omega_{n}$ are smooth domains such that $\Omega_{n} \rightarrow \Omega$ as $n \rightarrow \infty$. Then $\lambda_{1}^{+}\left(\Omega_{n}\right) \rightarrow \lambda_{1}^{+}(\Omega)$, and $\lambda_{1}^{-}\left(\Omega_{n}\right) \rightarrow \lambda_{1}^{-}(\Omega)$.

Proof. The two limits are proved in the same way, so let us prove the first. We already know that there exist positive eigenfunctions $\varphi_{1}^{(n)}, \varphi_{1}^{+}$for $F$ in $\Omega_{n}, \Omega$. If $\Omega_{n+1} \subset \Omega_{n} \subset \Omega$ for each $n$, the proposition is proved through exactly the same argument as the one in the proof of Theorem 4.2.

Suppose now $\Omega \subset \Omega_{n} \subset \Omega_{n+1}$ for each $n$. Then $\lambda_{1}^{+}\left(\Omega_{n}\right)$ is nondecreasing and bounded above by $\lambda_{1}^{+}(\Omega)$. Again through the same argument as the one in the proof of Theorem 4.2 we can show that $\varphi_{1}^{(n)}$ is uniformly bounded in $L^{\infty}(\Omega)$, and converges to a positive function $\varphi$ in $W_{\text {loc }}^{2, p}(\Omega)$. By Proposition 4.9 $\left\{\varphi_{1}^{(n)}\right\}$ is equicontinuous in $\bar{\Omega}$, and hence converges (up to a subsequence) to a function $\varphi$ in $C(\bar{\Omega})$. Then $\varphi=t \varphi_{1}^{+}$is a consequence of Corollary 4.1.

Finally, for any given sequence $\Omega_{n}$, such that $\Omega_{n} \rightarrow \Omega$, we can find sequences $\Omega_{n}^{\prime}, \Omega_{n}^{\prime \prime}$ such that $\Omega_{n}^{\prime}$ is increasing, $\Omega_{n}^{\prime \prime}$ is decreasing, $\Omega_{n}^{\prime} \subset \Omega_{n}$, $\Omega_{n}^{\prime} \subset \Omega, \Omega_{n} \subset \Omega_{n}^{\prime \prime}$, and $\Omega \subset \Omega_{n}^{\prime \prime}$. Proposition 4.10 then follows from what we already proved.

Lemma 4.5 Assume $\lambda_{1}^{+}(\Omega)>0$. Then there exists a function $v$ such that $v \in E_{p}, \forall p<\infty$, satisfying

$$
F\left(D^{2} v, D v, v, x\right) \leq 0 \text { in } \Omega, \quad 1 \leq v \leq C \text { in } \Omega
$$

where the constant $C$ depends on $\lambda, \Lambda, N, \Omega, \gamma, \delta$, and $\lambda_{1}^{+}$. Respectively, if $\lambda_{1}^{-}>0$ then there exists a function $w \in E_{p}$ such that $-C \leq w \leq-1$ and $F\left(D^{2} w, D w, w, x\right) \geq 0$.

Proof. First, by Proposition 4.9 there exists a neighbourhood of $\partial \Omega$, depending only on $N, \lambda, \Lambda, \gamma, \delta$ and $\lambda_{1}^{+}$(or $\lambda_{1}^{-}$), such that $\varphi_{1}^{+}$(or $\varphi_{1}^{-}$) attains its maximum (minimum) outside this neighbourhood.

Next, we use the idea of the proof of Proposition 6.1 in [BNV]. Choose a compact set $K \subset \Omega$ such that $\varphi_{1}^{+}$attains its maximum (set to 1 ) in $K$, and such that $|\Omega \backslash K| \leq \varepsilon=(2 \delta B)^{-N}$ where $B$ is the constant in Theorem 3.1. We solve the problem

$$
F\left(D^{2} w, D w, 0, x\right)=\left\{\begin{array}{ccc}
-2 \delta & \text { in } & \Omega \backslash K \\
0 & \text { in } & K
\end{array}\right.
$$

and $w=0$ on $\partial \Omega$ (see Theorem 3.4). By Theorem 3.1 we have

$$
0<w \leq B 2 \delta \varepsilon^{1 / N}=1 \quad \text { in } \Omega
$$


Then, by $\left(H_{1}\right)$,

$$
\begin{aligned}
F\left(D^{2}(w+1), D(w+1), w+1, x\right) & \leq F(0,0, w+1, x)+F\left(D^{2} w, D w, 0, x\right) \\
& \leq \delta(w+1)-2 \delta \leq 0 \quad \text { in } \Omega \backslash K .
\end{aligned}
$$

By the Harnack inequality (Theorem 3.6), we know that $\varphi_{1}^{+} \geq \eta$ on $K$, for some $\eta>0$ which depends on the appropriate quantities. Set $A=\frac{2 \delta}{\lambda_{1}^{+} \eta}$ and $v=1+w+A \varphi_{1}^{+}$. Then $1 \leq v \leq 2+A=: C$.

Further, by $\left(H_{1}\right)$,

$$
\begin{aligned}
& F\left(D^{2} v, D v, v, x\right) \leq F\left(D^{2}(w+1), D(w+1), w+1, x\right)-A \lambda_{1}^{+} \varphi_{1}^{+}
\end{aligned}
$$

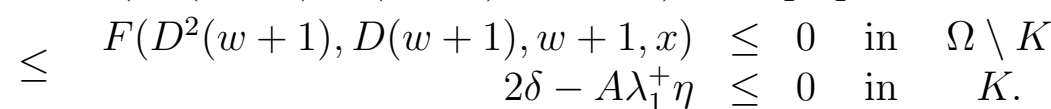

To get the corresponding statement when $\lambda_{1}^{-}>0$ we can repeat the same argument, reversing signs and inequalities where appropriate.

Note that we cannot directly infer from the proof of Lemma 4.5 that the function $v$ is in $W^{2, p}(\Omega)$, because of unavailability of global $W^{2, p}$-estimates for fully nonlinear equations. For completeness (at least we are unaware of any reference dealing with that question), we use the following construction. Set

$$
\Omega_{d}=\left\{x \in \mathbb{R}^{N} \mid \operatorname{dist}(x, \Omega)<d\right\} .
$$

Then if $d$ is small enough (say $d \leq d_{0}$, depending only on the shape of $\Omega$ ) $\Omega_{d}$ is smooth, and by $\left(H_{2}\right)$ we can continuously extend the function $F(M, 0,0, x)$ in $\mathcal{S}_{N} \times \Omega_{d}$. Define $\widehat{F}=F$ if $x \in \Omega$, and $\widehat{F}(M, p, u, x)=F(M, 0,0, x)$ for each $x \notin \Omega$. The extended operator $\widehat{F}$ has the same properties as $F$, namely, is convex in $(M, p, u)$ and satisfies $\left(H_{0}\right),\left(H_{1}\right),\left(H_{2}\right)$.

Proposition 4.11 We have $\lambda_{1}^{+}(\Omega)=\widetilde{\lambda}_{1}^{+}(\Omega)$.

Proof. Fix $d \leq d_{0}$. For each $\varepsilon>0$ we can apply Lemma 4.5 to the operator $\widehat{F}+\left(\lambda_{1}^{+}\left(\widehat{F}, \Omega_{d}\right)-\varepsilon\right)$ in the domain $\Omega_{d}$, which yields $\widetilde{\lambda}_{1}^{+}(F, \Omega) \geq$ $\lambda_{1}^{+}\left(\widehat{F}, \Omega_{d}\right)-\varepsilon$, by the definitions. By letting first $\varepsilon \rightarrow 0$, then $d \rightarrow 0$ and by using Proposition 4.10 , we get $\widetilde{\lambda}_{1}^{+}(F, \Omega) \geq \lambda_{1}^{+}(F, \Omega)$. Since by definition $\widetilde{\lambda}_{1}^{+} \leq \lambda_{1}^{+}$, Proposition 4.11 is proved.

Proposition 4.12 Assume $\lambda_{1}^{+}(\Omega)>0$. Then there exists a function $v$ such that $v \in W^{2, p}(\Omega)$, for all $p<\infty$, satisfying

$$
F\left(D^{2} v, D v, v, x\right) \leq 0 \quad \text { in } \Omega, \quad v \geq 1 \text { in } \Omega, \quad\|v\|_{W^{2, p}(\Omega)} \leq C,
$$

where the constant $C$ depends on $p, \lambda, \Lambda, N, \Omega, \gamma, \delta$, and $\lambda_{1}^{+}$. Respectively, if $\lambda_{1}^{-}>0$ then there exists a function $w \in W^{2, p}(\Omega)$ such that $-C \leq w \leq-1$, $F\left(D^{2} w, D w, w, x\right) \geq 0$, and $\|w\|_{W^{2, p}(\Omega)} \leq C$. 
Proof. In view of the previous proposition we only need to prove the bound on the $W^{2, p}$-norm. We are going to show that there exists $d_{0}>0$, depending on $\lambda, \Lambda, N, \Omega, \gamma, \delta$ and $\lambda_{1}^{+}$, such that $\lambda_{1}^{+}\left(\Omega_{d_{0}}\right)>0$. The result will then follow from Lemma 4.5 and the interior $W^{2, p}$-estimates (Theorem 3.3).

Take the function $v$ we constructed in Lemma 4.5. Looking at its construction, $v=1+w+A \varphi_{1}^{+}$(see the proof of Lemma 4.5), we have $v \equiv 1$ on $\partial \Omega$, and $v(x) \leq 1+k_{0} \operatorname{dist}(x, \partial \Omega)$, by Proposition 4.9 applied to $w$ and $\varphi_{1}^{+}$. Here $k_{0}$ depends on the right quantities.

We extend $v$ outside $\Omega$ in the following way : given $d_{0}$ such that the distance function to the boundary of $\Omega$ is smooth in $\Omega_{d_{0}} \backslash \bar{\Omega}$ (we refer to [GT], Chapter 14.6, for properties of the distance function), for each $y \in \Omega_{d_{0}} \backslash \bar{\Omega}$ we set

$$
v(y)=1-\left(k_{0}+1\right) d(y)-\bar{C} d^{2}(y), \quad \text { where } 0 \leq d(y)=\operatorname{dist}(y, \partial \Omega) \leq d_{0} .
$$

Of course $y=x+d(y) n(x)$, where $n(x), x \in \partial \Omega$, is the unit exterior normal to $\partial \Omega$, and $x$ is the point where $\operatorname{dist}(y, \partial \Omega)$ is attained. The extended function $v$ is clearly continuous in $\Omega_{d_{0}}$.

Claim. We can choose $d_{0}$ sufficiently small, and $\bar{C}$ sufficiently large, depending only on the appropriate quantities, such that

$$
\mathcal{M}_{\lambda, \Lambda}^{+}\left(D^{2} v\right) \leq-1 \quad \text { in } \Omega_{d_{0}} \backslash \bar{\Omega} .
$$

Proof of the Claim. Fix $y$ and a principal coordinate system at $x$ (see [GT], page 354). Then

$$
D^{2} d(y)=\operatorname{diag}\left(\frac{\kappa_{1}}{1-\kappa_{1} d(y)}, \ldots, \frac{\kappa_{n-1}}{1-\kappa_{n-1} d(y)}, 0\right)
$$

where $\kappa_{i}=\kappa_{i}(x)$ are the principal curvatures of $\partial \Omega$ at $x$, see Lemma 14.17 in $[\mathrm{GT}]$. Clearly there exists a constant $\kappa$ depending only on $\Omega$ such that $\left|\kappa_{i}(x)\right| \leq \kappa$ for all $i$ and all $x \in \partial \Omega$. Take $d_{0}$ so small that $\kappa d_{0} \leq \frac{1}{2}$.

Since $|D d|=1$ we have $D d \otimes D d(y)=\operatorname{diag}(0, \ldots, 0,1)$, in the same coordinate system. Now, by using

$$
D^{2}\left(d^{2}\right)=2 d D^{2} d+2 D d \otimes D d,
$$

we see $-2 \bar{C}$ is an eigenvalue of $D^{2} v$, while each of the other $N-1$ eigenvalues of $D^{2} v$ is bounded by $2\left(\left(k_{0}+1\right) \kappa+2 \kappa d \bar{C}\right)$. This implies

$$
\mathcal{M}_{\lambda, \Lambda}^{+}\left(D^{2} v\right) \leq 2 \Lambda(N-1)\left(\left(k_{0}+1\right) \kappa+2 \kappa d \bar{C}\right)-2 \lambda \bar{C}
$$

Taking $d_{0}<\frac{\lambda}{4 \kappa \Lambda N}$ and $\bar{C}=\frac{2 \Lambda N \kappa\left(k_{0}+1\right)+1}{\lambda}$ yields the claim. 
Therefore, by the way we extended $F$,

$$
F\left(D^{2} v, D v, v, x\right)=F\left(D^{2} v, 0,0, x\right) \leq \mathcal{M}_{\lambda, \Lambda}^{+}\left(D^{2} v\right) \leq-1 \quad \text { in } \Omega_{d_{0}} \backslash \bar{\Omega}
$$

with the choice of $d_{0}$ we made above. So we already know that we have $F\left(D^{2} v, D v, v, x\right) \leq 0$ in $\Omega \cup\left(\Omega_{d_{0}} \backslash \bar{\Omega}\right)$. Finally, the function $v$ is a viscosity solution of $F\left(D^{2} v, D v, v, x\right) \leq 0$ in $\Omega_{d_{0}}$ - this follows from the fact that we have constructed $v$ to be sufficiently "steep" outside $\Omega$, namely

$$
v(x) \leq\left\{\begin{array}{lll}
1+k_{0} d(x, \partial \Omega) & \text { for } & x \in \Omega \\
1-\left(k_{0}+1\right) d(x, \partial \Omega) & \text { for } & x \notin \Omega
\end{array}\right.
$$

so no regular function can "touch from below" the graph of $v$ at a point on $\partial \Omega$. By the definition of $\lambda_{1}^{+}$we infer $\lambda_{1}^{+}\left(\Omega_{d}\right)>0$ for each $d<d_{0}$.

Getting the statement for $\lambda_{1}^{-}>0$ is again a matter of reversing signs and inequalities.

Proof of Theorem 1.4 Part (a) is a consequence of Theorem 4.1. Indeed, if $u$ is as in Theorem 1.4 (a) then $\lambda_{1}^{+} \geq 0$, by the definition of $\lambda_{1}^{+}$. If $\lambda_{1}^{+}=0$ then Theorem 4.1 with $v=\varphi_{1}^{+}$implies $u=t \varphi_{1}^{+}$.

Part (b) follows from Proposition 4.12.

\subsection{Conclusion. Proofs of Theorems 1.5-1.10}

Proof of Theorem 1.5 That $\lambda_{1}^{+}>0$ suffices for $(C P)$ follows from Propositions 4.4 and 4.11 .

On the other hand, if $\lambda_{1}^{+} \leq 0$ then $\varphi_{1}^{+}$is a counterexample to the maximum principle, since then $F\left(D^{2} \varphi_{1}^{+}, D \varphi_{1}^{+}, \varphi_{1}^{+}, x\right) \geq 0$ in $\Omega, \varphi_{1}^{+}=0$ on $\partial \Omega$, but $\varphi_{1}^{+} \not \leq 0$ in $\Omega$.

Proof of Theorem 1.7 Theorem 1.7 is a consequence of Proposition 4.3 in which we take $\psi_{1}, \psi_{2}$ to be the functions $v, w$ constructed in Proposition 4.12 , in view of the imbedding $W^{2, p}(\Omega) \hookrightarrow C^{1, \alpha}(\Omega)$.

Proof of Theorem 1.6 Use Theorem 1.7 and note that if $\lambda_{1}^{-} \leq 0$ then $\varphi_{1}^{-}\left(\varphi_{1}^{-}<0\right.$ in $\left.\Omega\right)$ provides a counterexample to the one-sided maximum principle.

Proof of Theorem 1.8 The existence result follows from Propositions 4.5 and 4.11. The $W^{2, p}$ bound follows from Theorem 3.3 and Theorem 1.7.

Suppose for contradiction $\lambda_{1}^{+}=0$ and $\bar{u} \in C(\bar{\Omega})$ is a function such that $F\left(D^{2} \bar{u}, D \bar{u}, \bar{u}, x\right)=f \leq 0$ in $\Omega, \bar{u}=0$ on $\partial \Omega$. First, if $\bar{u} \geq 0$ in $\Omega$, then by Lemma $3.1 \bar{u}>0$ in $\Omega$ (note $\bar{u} \not \equiv 0$ since $f \not \equiv 0$ ). Then $u=\bar{u}$ and $v=\varphi_{1}^{+}$ satisfy the first set of inequalities in Theorem 4.1 , so $\bar{u}=t \varphi_{1}^{+}$, which yields a contradiction with $f \not \equiv 0$. 
Second, if $\bar{u}$ is negative somewhere, we have the second set of inequalities in Theorem 4.1, with $u=\varphi_{1}^{-}$and $v=\bar{u}$ (recall that $F\left(D^{2} \varphi_{1}^{-}, \ldots\right)=-\lambda_{1}^{-} \varphi_{1}^{-}$, $\varphi_{1}^{-}<0$ in $\Omega$, and note $\left.\lambda_{1}^{-} \geq \lambda_{1}^{+}=0\right)$. We get $f \geq 0$ and $f \leq 0$, a contradiction with $f \not \equiv 0$.

Finally, if $\lambda_{1}^{-}>0 \geq \lambda_{1}^{+}$then Theorem 1.6 and Lemma 3.1 imply $u>0$ or $u \equiv 0$ in $\Omega$, so we can conclude as in the previous two cases.

Proof of Theorem 1.9 As in the proof of Proposition 4.5, $\widetilde{\lambda}_{1}^{-}=\lambda_{1}^{-}>0$ implies the existence of a negative subsolution $u_{0}$, provided $f \in C(\bar{\Omega})$. On the other hand, $f \geq 0$ implies that $v_{0} \equiv 0$ is a supersolution. Then Lemma 4.3 shows Theorem 1.9 holds in case the right-hand side $f$ is in $C(\bar{\Omega})$.

If $f \in L^{p}$ is not in $C(\bar{\Omega})$, we take a sequence $f_{n} \in C(\bar{\Omega}), f_{n} \geq 0$, which tends to $f$ in the $L^{p}$-norm. By what we already proved, there exists a solution $u_{n} \in E_{p}, u_{n} \leq 0$, of $F\left(D^{2} u_{n}, D u_{n}, u_{n}, x\right)=f_{n}$, and $u_{n}=0$ on $\partial \Omega$. Note that we cannot use the same approximation argument as in the proof of Proposition 4.5, since the ABP inequality does not hold. However, the halfABP inequality which still holds under $\lambda_{1}^{-}>0$ (see Theorem 1.7) implies that $u_{n}$ is uniformly bounded in $\Omega$ (recall $u_{n} \leq 0$ ). Then by interior $W^{2, p}$ estimates $u_{n}$ converges in $W_{\text {loc }}^{2, p}$ to a solution $u$ of $F\left(D^{2} u, D u, u, x\right)=f$. It only remains to show that $u=0$ on $\partial \Omega$ and $u \in C(\bar{\Omega})$.

Take again a compact subset $K \subset \Omega$ such that $|\Omega \backslash K| \leq(2 \delta B)^{-N}$, where $B$ is the constant in Theorem 3.1. We already know that $u_{n} \rightrightarrows u$ in $K$. Now the inequality

$F\left(D^{2}\left(u_{n}-u_{m}\right), D\left(u_{n}-u_{m}\right), u_{n}-u_{m}, x\right)-\delta\left(u_{n}-u_{m}\right) \geq f_{n}-f_{m}-\delta\left(u_{n}-u_{m}\right)$,

(and the same with $m$ and $n$ interchanged), together with Theorem 3.1, yield

$$
\sup _{\Omega \backslash K}\left|u_{n}-u_{m}\right| \leq \sup _{\partial K}\left|u_{n}-u_{m}\right|+B\left\|f_{n}-f_{m}\right\|_{L^{p}(\Omega)}+\frac{1}{2} \sup _{\Omega \backslash K}\left|u_{n}-u_{m}\right| .
$$

This implies that $\left\{u_{n}\right\}$ is a Cauchy sequence in $C(\overline{\Omega \backslash K})$ and so converges uniformly in this set.

Proof of Theorem 1.10 We are going to use the results for proper operators obtained in [CKLS].

First, suppose $H$ and $f$ are continuous in all their variables. Let $\phi=v$ be the function constructed in Proposition 4.12. Then, as we already showed in the proof of Proposition 4.3, the operator $H_{\phi}$ satisfies hypotheses (1.2) and (1.3) in [CKLS] (we define $H_{\phi}$ as in (4.17) with $F$ replaced by $H$ ). Hence Theorem 1.10 follows from Theorem 1.1 in [CKLS] - note that if $\bar{u}$ is a solution of $H_{\phi}\left(D^{2} \bar{u}, D \bar{u}, \bar{u}, x\right)=f$, then $u=\phi \bar{u}$ is a solution of $H\left(D^{2} u, D u, u, x\right)=f$. 
Next, if $H$ is only measurable, we smooth out $G, H$, and $\mathrm{F}$ as in the proof of Theorem 4.1 in [CKLS]

$$
F_{\varepsilon}(M, p, u, x)=\frac{1}{\varepsilon^{n}} \int_{\mathbb{R}^{N}} \eta\left(\frac{x-y}{\varepsilon}\right) F(M, p, u, y) d y, \quad \text { same for } H_{\varepsilon}, G_{\varepsilon},
$$

where $\eta \geq 0$ has compact support and mass 1 . Now, for fixed $\varepsilon$, the operators $H_{\varepsilon}, G_{\varepsilon}, F_{\varepsilon}$ satisfy all the hypotheses we have made on $H, G, F$, with the same constants $\lambda, \Lambda, \gamma, \delta$.

In particular, $F_{\varepsilon}$ also possesses a principal eigenvalue $\lambda_{1}^{+}\left(F_{\varepsilon}, \Omega\right)$. Claim. $\lambda_{1}^{+}\left(F_{\varepsilon}\right) \rightarrow \lambda_{1}^{+}(F)$, as $\varepsilon \rightarrow 0$.

Proof of the Claim. By the bounds on the first eigenvalue that we have already proved (Proposition 4.2) $\left\{\lambda_{1}^{+}\left(F_{\varepsilon}\right)\right\}$ is bounded in $\varepsilon$. Fix a subsequence of $\left\{\lambda_{1}^{+}\left(F_{\varepsilon}\right)\right\}$ and let $\mu$ be the limit of some subsequence of this subsequence. We are going to show that $\mu=\lambda_{1}^{+}(F)$. Let $\varphi_{1, \varepsilon}^{+}$be the first eigenfunctions of $F_{\varepsilon}$ normalized so that $\varphi_{1, \varepsilon}^{+}\left(x_{0}\right)=1$ for a fixed point $x_{0} \in \Omega$. Then $\left\{\varphi_{1, \varepsilon}\right\}$ is uniformly bounded in the $L^{\infty}$-norm, by Theorem 1.1. Hypothesis $\left(H_{1}\right)$ and the equation satisfied by $\varphi_{1, \varepsilon}$ then imply

$$
\mathcal{M}_{\lambda, \Lambda}^{+}\left(D^{2} \varphi_{1, \varepsilon}\right)+\gamma\left|D \varphi_{1, \varepsilon}\right| \geq-C, \quad \mathcal{M}_{\lambda, \Lambda}^{-}\left(D^{2} \varphi_{1, \varepsilon}\right)-\gamma\left|D \varphi_{1, \varepsilon}\right| \leq C,
$$

where $C$ is independent of $\varepsilon$. Hence, by Proposition 4.2 in [CKLS] (see also the end of the proof of Theorem 4.1 in this paper), a subsequence of $\left\{\varphi_{1, \varepsilon}\right\}$ converges uniformly in $\bar{\Omega}$ to a positive function $\varphi \in C(\bar{\Omega})$, which solves (see also Theorem 3.8 in $[\mathrm{CCKS}]$ )

$$
F\left(D^{2} \varphi, D \varphi, \varphi, x\right)=-\mu \varphi \text { in } \Omega, \quad \text { and } \quad u=0 \text { on } \partial \Omega .
$$

So $\mu=\lambda_{1}^{+}(F, \Omega)$, by Theorem 1.1 .

Hence, if $\varepsilon$ is small enough, we have $\lambda_{1}^{+}\left(F_{\varepsilon}\right)>\frac{1}{2} \lambda_{1}^{+}(F)>0$.

We take a sequence $f_{n} \in C(\bar{\Omega})$ which converges to $f$ in $L^{p}$. By what we already proved, if $\varepsilon$ is small enough, there exists a solution $u_{\varepsilon, n}$ of

$$
H_{\varepsilon}\left(D^{2} u_{\varepsilon, n}, D u_{\varepsilon, n}, u_{\varepsilon, n}, x\right)=f_{n},
$$

and $u_{\varepsilon, n}=0$ on $\partial \Omega$. Since $H$ satisfies $\left(D_{F}\right)$ this implies

$$
\begin{gathered}
F_{\varepsilon}\left(D^{2} u_{\varepsilon, n}, D u_{\varepsilon, n}, u_{\varepsilon, n}, x\right) \geq f_{n}, \quad \text { and } \\
F_{\varepsilon}\left(D^{2}\left(-u_{\varepsilon, n}\right), D\left(-u_{\varepsilon, n}\right),-u_{\varepsilon, n}, x\right) \geq-f_{n}
\end{gathered}
$$

in $\Omega$. By the ABP inequality (Theorem 1.7) $u_{\varepsilon, n}$ is uniformly bounded in $\epsilon$ and $n$, so we can finish the proof of Theorem 1.10 through the same argument as the one used to end the proof of Theorem 4.1 in [CKLS].

The last statement in Theorem 1.10 is a consequence of Theorem 3.3. 


\section{General bounded domains}

In $[\mathrm{BNV}]$ Berestycki, Nirenberg and Varadhan proved the existence of a principal eigenvalue and a principal eigenfunction of linear elliptic operators with bounded (and continuous second-order) coefficients in arbitrary bounded domains. As noted in the introduction, once we have proved our theorems in smooth domains, it is not difficult to adapt some arguments from [BNV] in order to show that most of our results extend to general domains. In this section we make several remarks concerning these extensions, leaving the details to the interested reader.

So suppose $\Omega$ is just bounded, $F$ satisfies $\left(H_{0}\right)-\left(H_{1}\right)$, and $F(M, 0,0, x)$ is continuous in $\mathcal{S}_{N} \times \Omega$. Similarly to Section 3 in [BNV] we use the positive function $\bar{u}_{0}$ defined as the limit of the solutions of $F\left(D^{2} u, D u, 0, x\right)=-1$ in smooth subdomains of $\Omega$ which converge to $\Omega$. Note that the negative function $\underline{u}_{0}$ defined as the limit of the solutions of $F\left(D^{2} u, D u, 0, x\right)=1$ satisfies $0<-\underline{u}_{0} \leq \bar{u}_{0}$, by the assumptions on $F$.

The functions we consider are not continuous up to the boundary, and the Dirichlet boundary condition $u=0$ is to be replaced in all results by $u \stackrel{\bar{u}_{0}}{=} 0$, that is, $u\left(x_{j}\right) \rightarrow 0$ whenever $x_{j} \rightarrow \partial \Omega$ and $\bar{u}_{0}\left(x_{j}\right) \rightarrow 0$. Inequalities $u \leq v$ on $\partial \Omega$ are to be replaced by $\lim \sup \left(u\left(x_{j}\right)-v\left(x_{j}\right)\right) \leq 0$ whenever $x_{j} \rightarrow \partial \Omega$ and $\bar{u}_{0}\left(x_{j}\right) \rightarrow 0$. In defining the comparison principle one has to explicitly state that the functions involved are bounded (see [BNV] for an example showing that this is unavoidable). The space $E_{p}$ has to be replaced by $W_{\text {loc }}^{2, p} \cap L^{\infty}(\Omega)$.

Then Theorem 1.1 is proved through the same approximation argument as the one used in the proof of Theorem 4.2, since we already know eigenvalues exist in the smooth subdomains. Theorem 1.2 and all other results following from Theorem 4.1 do not change. Theorem 1.4 (b) has to be replaced by the result in Lemma 4.5, which remains true (but Proposition 4.12 no longer holds). The results on solvability of the Dirichlet problem and the ABP inequality are proved by an adaptation of the arguments in Section 6 of $[\mathrm{BNV}]$, by using at the appropriate places the fact that the Dirichlet problem is already known to be solvable in smooth subdomains of $\Omega$.

\section{$6 \quad$ Proof of Theorem 1.3}

It follows from Theorem 1.8 that $u \equiv 0$ is the unique solution of (1.4), when $\lambda<\lambda_{1}^{+}$. If $\lambda<\lambda_{1}^{-}$and $u \not \equiv 0$ is a solution of (1.4) then it follows from Theorem 1.6 and the strong maximum principle that $u>0$ in $\Omega$. Then $\lambda=\lambda_{1}^{+}$, by the last statement in Theorem 1.1.

Finally, suppose there exists a sequence $\lambda_{n} \searrow \lambda_{1}^{-}$such that problem (1.4) 
with $\lambda=\lambda_{n}$ has a solution $u_{n} \not \equiv 0$. We normalize $u_{n}$ so that $\left\|u_{n}\right\|_{L^{\infty}(\Omega)}=1$. $\operatorname{By}\left(H_{1}\right)$

$$
\mathcal{M}_{\lambda, \Lambda}^{+}\left(D^{2} u_{n}\right)+\gamma\left|D u_{n}\right| \geq-C, \quad \mathcal{M}_{\lambda, \Lambda}^{-}\left(D^{2} u_{n}\right)-\gamma\left|D u_{n}\right| \leq C,
$$

and again from Proposition 4.2 in [CKLS] and Theorem 3.8 in [CCKS] it follows that $u_{n}$ converges uniformly in $\bar{\Omega}$ to a nontrivial solution of (1.4) with $\lambda=\lambda_{1}^{-}$, in other words, to $\varphi_{1}^{-}$. However, $u_{n}$ changes sign, by Theorem 1.1, so we can take a connected component $\Omega_{n}$ of $\left\{u_{n}>0\right\}$. Then, by what we have already proved for non-regular domains, $\lambda_{1}^{+}\left(F, \Omega_{n}\right)=\lambda_{n} \rightarrow \lambda_{1}^{-}$. On the other hand $\varphi_{1}^{-}<0$ implies $\Omega_{n} \rightarrow \emptyset$, hence $\lambda_{1}^{+}\left(F, \Omega_{n}\right) \rightarrow \infty$ by Proposition 4.8, which is a contradiction.

\section{References}

[BD] M. Bardi, F. Da Lio, On the strong maximum principle for fully nonlinear degenerate elliptic equations. Arch. Math. (Basel) 73 (1999), 276-285.

[Be] H. Berestycki, On some nonlinear Sturm-Liouville problems, J. Diff. Eq. 26 (1977), 375-390.

[BNV] H. Berestycki, L. Nirenberg, S.R.S. Varadhan, The principal eigenvalue and maximum principle for second order elliptic operators in general domains, Comm. Pure Appl. Math. 47(1) (1994), 47-92.

[BD1] I. Birindelli, F. Demengel, First eigenvalue and maximum principle for fully nonlinear singular operators. Adv. Diff. Eq. 11 (1) (2006), 91-119.

[Bu] J. Busca, Existence results for Bellman equations and maximum principles in unbounded domains. Comm. Part. Diff. Eq. 24 (11-12) (1999), 2023-2042.

[BEQ] J. Busca, M. Esteban, A. Quaas, Nonlinear eigenvalues and bifurcation problems for Pucci's operator, Ann. Inst. H. Poincaré, Anal. Nonl. 22 (2) (2005), 187-206.

[BS] J. Busca, B. Sirakov, Harnack type estimates for nonlinear elliptic systems and applications, Ann. Inst. H. Poincare, Anal. Nonl. 21 (5) (2004), 543-590.

[C] L.A. Caffarelli, Interior estimates for fully nonlinear elliptic equations, Ann. of Math. 130 (1989), 189-213. 
[CC] L.A. Caffarelli, X. Cabre, Fully Nonlinear Elliptic Equations, A.M.S. Coll. Publ. Vol 43, Providence (1995).

[CCKS] L.A. Caffarelli, M.G. Crandall, M.Kocan, A. Świech, On viscosity solutions of fully nonlinear equations with measurable ingredients, Comm. Pure Appl. Math 49 (1996), 365-397.

[CKLS] M.G. Crandall, M. Kocan, P.L. Lions, A. Świech, Existence results for boundary problems for uniformly elliptic and parabolic fully nonlinear equations, Elec. J. Diff. Eq. 24 (1999), 1-20.

[CKS] M.G. Crandall, M. Kocan, A. Świech, $L^{p}$ theory for fully nonlinear uniformly parabolic equations, Comm. Part. Diff. Eq. 25, (11-12) (2000), 1997-2053.

[CIL] M.G. Crandall, H. Ishii, P.-L. Lions, User's guide to viscosity solutions of second-order partial differential equations, Bull. Amer. Math. Soc. 27 (1992) (1), 1-67.

[E] L.C. Evans, Classical solutions of fully nonlinear, convex, second-order elliptic equations, Comm. Pure Appl. Math. 25 (1982), 333-363.

[FQ] P. Felmer, A. Quaas, Positive solutions to 'semilinear' equation involving the Pucci's operator, J. Diff Eq. 199 (2) (2004), 376-393.

[GT] D. Gilbarg, N.S. Trudinger, Elliptic Partial Differential Equations of Second Order, 2nd edition, Springer Verlag.

[HNR] F. Hamel, N. Nadirashvili, E. Russ, A Faber-Krahn inequality with drift, preprint. F. Hamel, N. Nadirashvili, E. Russ, An isoperimetric inequality for the principal eigenvalue of the Laplacian with drift, C. R. Acad. Sci. Paris Ser. I 340 (2005), 347-352.

[JS] R. Jensen, A. Swiech, Uniqueness and existence of maximal and minimal solutions of fully nonlinear PDE, Comm. Pure Appl. Anal. 4 (1) (2005), 187-195.

[K] N.V. Krylov, Nonlinear elliptic and parabolic equations of second order. Mathematics and its Applications, Reidel, 1987.

[L1] P.L. Lions, Bifurcation and optimal stochastic control, Nonl. Anal. Th. and Appl. 7(2) (1983), 177-207.

[L2] P.L. Lions, Résolution analytique des problèmes de Bellman-Dirichlet, Acta Math. 146 (1981), 151-166. 
[Q] A. Quaas, Existence of Positive Solutions to a 'semilinear' equation involving the Pucci's operator in a convex domain, Diff. Int. Eq. 17 (2004), 481-494.

[S] A. Świech, $W^{1, p}$-estimates for solutions of fully nonlinear uniformly elliptic equations, Adv. Diff. Eq. 2(6) (1997), 1005-1027.

[W] L. Wang, On the regularity theory of fully nonlinear parabolic equations: I, Comm. Pure Appl. Math. 45 (1) (1992), 27-76. 